\title{
How to discover QCD Instantons at the LHC
}

\author{
Simone Amoroso ${ }^{1}$ (D) Deepak $\operatorname{Kar}^{2}$, Matthias Schott ${ }^{3}$, a \\ ${ }^{1}$ DESY, Hamburg, Germany \\ ${ }^{2}$ University of Witwatersrand, Johannesburg, South Africa \\ ${ }^{3}$ Johannes Gutenberg-University, Mainz, Germany
}

Received: 8 June 2021 / Accepted: 5 July 2021 / Published online: 17 July 2021

(C) The Author(s) 2021

\begin{abstract}
The Standard Model of particle physics predicts the existence of quantum tunnelling processes across topological inequivalent vacua, commonly known as Instantons. In Quantum Chromodynamics, these Instantons play a fundamental role in explaining much of the theory long-distance behaviour. However, they have not yet been observed experimentally. Their direct observation would mark a breakthrough in modern particle physics, shedding light on our fundamental understanding of the non perturbative dynamics in the Standard Model. Recently, new calculations for QCD Instanton processes in proton-proton collisions became public, suggesting sizeable cross sections as well as possible experimental signatures at the LHC. In this work, we explore possible analysis strategies for the LHC experiments to discover small-size QCD Instanton induced processes. Moreover, we derive a first limit on the Instanton production cross section using published data of Minimum Bias processes at $\sqrt{s}=13 \mathrm{TeV}$ at the LHC.
\end{abstract}

\section{Contents}

1 Introduction . . . . . . . . . . . . . 1

2 QCD Instanton processes at the LHC . . . . . . 3

2.1 Production of the instanton pseudo-particle in proton-proton collisions . . . . . . . . 3

2.2 Decay of the instanton pseudo-particle and experimental observables . . . . . . . . . 4

3 MC samples, detector simulation and experimental observables . . . . . . . . . . . . . . 4

3.1 Soft QCD processes . . . . . . . . . . . . . . 4

3.2 Hard QCD and other processes . . . . . . . . 5

3.3 Signal simulation . . . . . . . . . . . 6

3.4 Experimental observables . . . . . . . . . . . 6

4 Search strategies . . . . . . . . . . . . 8

4.1 Very low instanton masses: the soft QCD regime 8

a e-mail: matthias.schott@cern.ch (corresponding author)
4.2 Medium instanton masses: the hard QCD regime 10

4.3 High instanton masses: the top quark regime . . 12

5 Limits on instanton processes in proton-proton col-

lisions . . . . . . . . . . . . . . . 15

5.1 How to mimic QCD instanton signatures . . . 15

5.2 LHC sensitivity projections . . . . . . . . 15

5.3 First limit on instanton processes from protonproton collision data . . . . . . . . 17

6 Conclusion . . . . . . . . . . . . . 18

References . . . . . . . . . . . . . . . . 18

\section{Introduction}

Yang-Mills theories [1], embedded in the Standard Model (SM) of particle physics, form the basis of our understanding of the strong and electroweak interactions. The beauty and success of the SM lies in its predictive power, achieved in the weakly coupled regime by calculations based on perturbative approaches. Perturbation theory, developed in order to describe hadron collisions at high energies, relies on this small size of the strong coupling constant at high momentum transfer and short distance. The study of Quantum Chromodynamics (QCD) in the perturbative regime has seen tremendous advancements in the last decades. Hard scattering cross sections have been calculated up to the third order in the strong coupling [2,3], and perturbative QCD predictions have been verified to incredible precision over many orders of magnitude of momentum transfer at high energy colliders [4]. At the same time a fundamental understanding of YangMills theories in the strongly coupled limit is still lacking, and remains one of the biggest challenges for particle physics to date.

Yang-Mills theories exhibit a rich and non-trivial vacuum structure. In particular, they admit semi-classical solutions corresponding to fluctuations of the gauge fields across topologically non-equivalent vacua, known as Instantons [5]. These inherently non-perturbative phenomena are of great 
theoretical interest and have been linked to many fundamental aspects of QCD (introductory reviews on the physics of instantons can be found in [6-9]). Even though Instanton processes are a core prediction of the SM, they have never been experimentally observed.

The Yang-Mills vacuum structure is depicted in Fig. 1, showing the energy density of the gauge field as a function of the Chern-Simons (or winding) number, $N_{C S}$, which characterises the topological charge of a system. Instantons describe tunnelling transitions in Minkowski spacetime between classically degenerate vacua, which only differ by their winding number by one unit, i.e. $\Delta N_{C S}=1$. Instanton solutions are not only localised in time, but also in space, i.e. they have a certain spatial extension. A second type of classical solutions, known as Sphalerons, corresponds to transitions from one vacuum by a half-integer winding number on top of the energy barrier (also shown in Fig. 1), where its static energy corresponds to the barrier height.

These tunnelling solutions differ significantly from ordinary solutions obtained in perturbation theory, where only field configurations corresponding to small changes of the vacuum field at $N_{C S}=0$ are accessible and minima which cannot be obtained by continuous transformation of the gauge field are ignored. Instanton and Sphaleron solutions provide crucial ingredients for an understanding of a number of nonperturbative issues in the SM. In the electroweak theory, Instanton and Sphaleron transitions are associated with $B+L$ violation. They become highly relevant at high temperatures $[7,10,11]$ and have a crucial impact on the evolution of the baryon and lepton asymmetries in the early universe (see also Ref. [12,13] for a review). In QCD, these topological solutions have been argued to play an important role in various long-distance aspects of the theory. They provide a possible solution to the axial $U(1)$ problem [14] and are associated with chiral symmetry breaking [15-17].

The height of the energy barrier between two vacua, called Sphaleron mass $M_{S p}$, in the electroweak theory is of the order $M_{S p} \sim \frac{\pi}{\alpha \rho_{\text {eff }}} \sim \pi \frac{M_{W}}{\alpha_{W}} \sim 10 \mathrm{TeV}$ [18], where $\alpha_{W}$ is the weak coupling constant and $\rho_{\mathrm{eff}}$ the effective Instanton size. As the energy barrier is below the LHC center of mass energy, one might think that electroweak Sphalerons should be produced, and could be observed at the LHC. The question whether manifestations of such topological fluctuations can be directly observed in high-energy experiments was already raised in the 1980s, in the context of the electroweak sector [19-22]. However, the difficulty of obtaining a coherent state makes these processes likely to remain unobservable at current and future colliders [23,24]. The situation is different for QCD Instanton processes, for which the energy barrier height, $M_{S p} \sim \frac{3 \pi}{4 \alpha_{s} \rho_{\text {eff }}} \sim Q$ [25], with $\alpha_{s}$ the strong coupling and the parameter $Q$ related to the energy scale of the underlying process, can be as low as a few $\mathrm{GeV}$. The phenomenology of Instanton production at colliders has been first developed in the context of Deep Inelastic ep Scattering at the HERA collider [26,27]. Searches for Instanton processes have been performed by the ZEUS and $\mathrm{H} 1 \mathrm{Col}-$ laborations [28-30] excluding the lower range of the predicted cross sections. It is then interesting to understand if these processes could also be measured at the LHC. Recent works have provided first calculations for LHC cross sections [31], and some discussions on the expected phenomenology [32]. In this work we explore in further details suitable analysis strategies at the LHC, in particular exploring the (relatively) small-size regime with Instanton masses of few tenths of a GeV, where the cross section is the highest. In this regime the challenge lies in finding suitable observables that, while retaining sensitivity to the soft decay products of the Instanton, can also be described to an acceptable level of accuracy by the phenomenological models of soft QCD activity.

The paper is structured as follows: In Sect. 2 we briefly provide a review on Instanton processes at the LHC, covering their expected production cross sections and experimental signature. This is followed by an overview of the Monte Carlo samples used in Sect. 3. Possible search strategies and the optimisation of the event selection are described in Sect. 4. The expected sensitivity of the proposed analysis, as well as first limit on QCD Instanton processes are presented in Sect. 5. The paper concludes in Sect. 6 .
Fig. 1 Instanton and Sphaleron processes in the topology of a Yang-Mills vacuum; energy density of the gauge field (y-axis) vs. winding number $N_{C S}$ (x-axis)

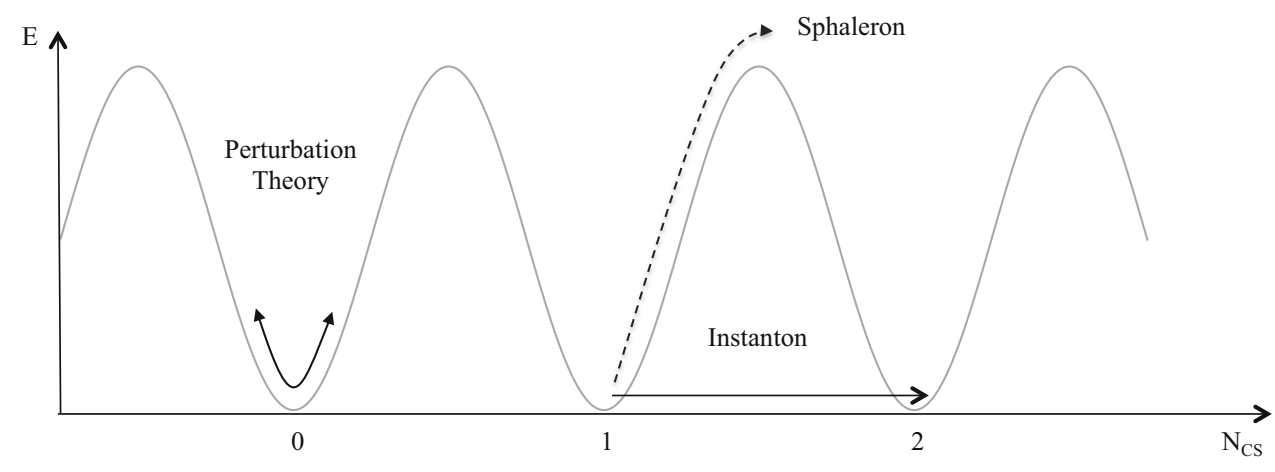


Fig. 2 Depiction of a QCD Instanton processes in electron-proton (left) and proton-proton (right) collisions, where an external scale parameter $Q^{\prime}$ is required
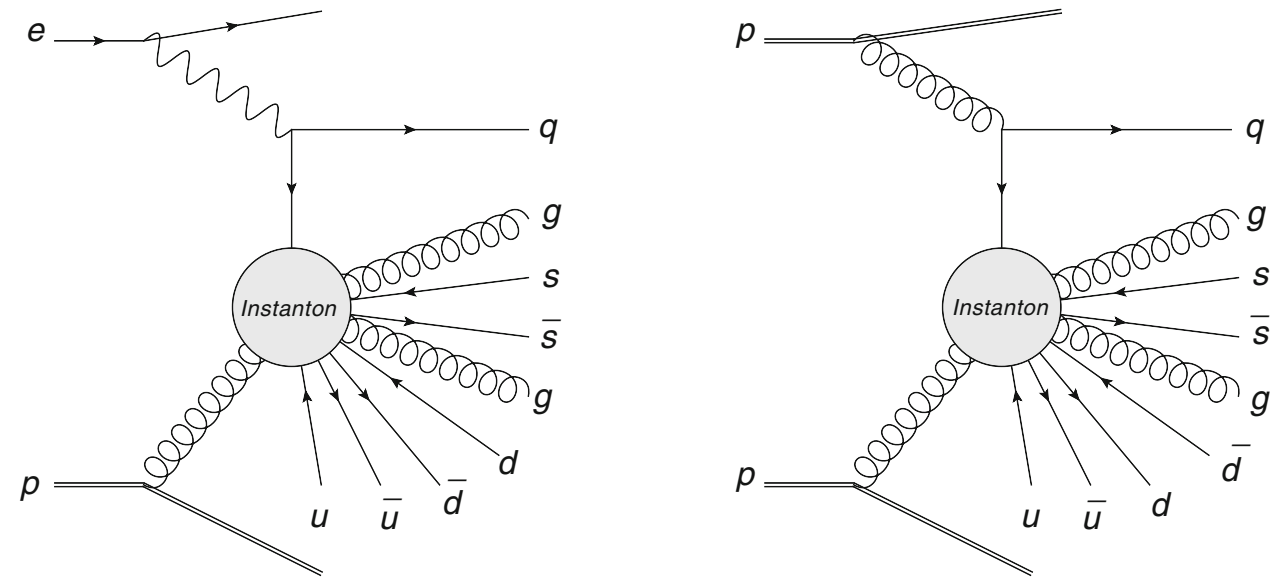

2 QCD Instanton processes at the LHC

2.1 Production of the instanton pseudo-particle in proton-proton collisions

Following the work of $[25,33]$ can write the inclusive partonic cross section of QCD Instanton-induced processes in Instanton perturbation theory as

$$
\begin{aligned}
\sigma_{\text {parton, parton }}^{(I)} & \int_{0}^{\infty} \int_{0}^{\infty} D(\rho) D(\bar{\rho}) d \rho d \bar{\rho} \cdot e^{-(\rho+\bar{\rho}) Q^{\prime}} \\
& \cdot \int \cdot e^{-\frac{4 \pi}{\alpha} \Omega\left(E / M_{I}\right)} \cdot \text { (further terms), }
\end{aligned}
$$

where $D(\rho)$ and $D(\bar{\rho})$ denote the Instanton and antiInstanton size distributions, $E$ the available energy of the process and $\Omega$ describes the Instanton anti-Instanton interaction, with $\Omega(x)=1$ for $x \rightarrow 0$ and $\Omega(x)=0$ for $x \rightarrow \infty$. The Instanton size distribution is proportional to $D(\rho) \sim \rho^{11-2 / 3 n_{f}-5}[15,25,34-36]$, thus an integral over $\rho$ would diverge. However, it was shown that the additional term $e^{-(\rho+\bar{\rho}) Q^{\prime}}$ has to be taken into account [25], where $Q^{\prime}$ describes a generic hard scale of the Instanton process. This form factor effect renders the $\rho$ integration convergent. In order to make reliable calculations of cross sections in QCD, Instanton perturbation theory [25] has to be applied. This requires the validity of the diluted gas approximation [25], which requires that the extensions of Instantons and antiInstantons are not overlapping. Therefore, the validity of Instanton perturbation theory requires Instantons to be sufficiently small, i.e. localised in space-time. In QCD, a generic hard scale $Q$ of the underlying process can be chosen, reducing the Instanton size and justifying the diluted gas approximation and the Instanton perturbation theory approach. In this scenario, the cross sections can become sizeable at high energies. The reason for the large cross section can be intuitively understood [37] by changing the picture from a tunnelling between vacua at $\mathrm{E}=0$ to that of the actual creation of a Sphaleron-like configuration [18] on top of the potential barrier of height. Therefore, in a naive (but not fully correct)

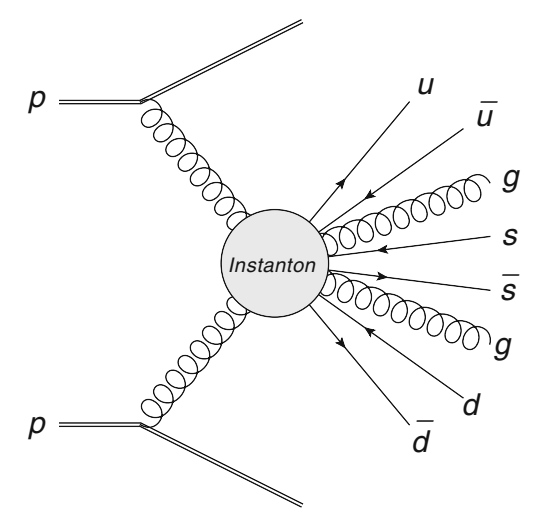

Fig. 3 Depiction of a QCD Instanton processes in proton-proton (right) collisions without the requirement of an external scale parameter $Q^{\prime}$

picture, the Instanton process can be interpreted as the creation and the decay of a Sphaleron pseudo-particle, where the pass of the pseudo-particle depends directly on the height of potential barrier.

In deep inelastic scattering processes, the hard scale $Q^{\prime}$ was defined by a highly virtual momentum transfer by a photon, emitted by the incoming electron, yielding a highly energetic final state quark in addition to the Instanton process, as shown in Fig. 2. This concept can be easily transferred to proton-proton collisions where the photon exchange is simply replaced by a gluon as shown in Fig. 2. A first calculation of the latter processes was completed in [32], but requiring the associated adronic jet to be sufficiently energetic lead to negligible cross sections at LHC energies.

An alternative strategy to calculate cross sections was also recently published [31]. Here, a second independent kinematic scale, as the DIS highly virtual momentum scale $Q$, is not required (Fig. 3). In this approach, only small Instantons contribute to the scattering processes in QCD and potentially problematic contributions of Instantons with large size are automatically cut-off by the inclusion of quantum effects due to interactions of the hard initial states that generate the factor $e^{-\alpha_{s} \rho^{2} s^{\prime}} \log s^{\prime}$. The latter reasoning was pre- 
sented in $1991[38,39]$ and provides a dynamical solution to the problem of IR divergences arising from Instantons of large scale-sizes in QCD. The scale invariance of the classical Yang-Mills theories is broken by those quantum effects which lead to a suppression of all but small Instantons with sizes $\rho \lesssim(10-30) / \sqrt{s^{\prime}}$. The characteristic QCD Instanton size is therefore inversely proportional to the centre-of-mass (CoM) energy of two colliding partons $\sqrt{s^{\prime}}$. Table 1 shows the proton-proton cross sections for Instanton processes at a center of mass energy of $13 \mathrm{TeV}$ for various choices of minimal values of $\sqrt{s_{\min }^{\prime}}$ as calculated in [31]. The cross section at $\sqrt{s_{\min }^{\prime}}=0 \mathrm{GeV}$ already contributes several percent to the total proton-proton cross section and at even lower values would saturate. This implies that the calculation breaks down at some small value of $\sqrt{s_{\text {min }}^{\prime}}$.

In the following it is assumed that the cross section estimate is reliable for $\sqrt{s_{\text {min }}^{\prime}}>20 \mathrm{GeV}$, keeping in mind that the uncertainty on this prediction could be of several order of magnitudes. The cross section dependence can be interpolated by a phenomenological formula $\sigma \sim e^{a \cdot e^{b \cdot x}+c \cdot x^{2}+d \cdot x+e}$, implying an exponential decrease vs. $\sqrt{s_{\text {min }}^{\prime}}$. In the pseudoparticle picture of the Instanton process, the available energy $\sqrt{s_{\min }^{\prime}}$ can be interpreted as the Instanton mass $m_{I}$. The production of Instantons is therefore not a resonant but a continuous processes, with large production rates for Instantons of low mass and small production rates for Instantons of high masses.

2.2 Decay of the instanton pseudo-particle and experimental observables

A QCD Instanton tunnelling process between $\Delta N_{C S}=1$ vacua leads to the creation of exactly one quark-antiquark pair of different chirality for each flavour, $N_{f}$ in association with a number $n_{g}$ of additional gluons. Following the approach of [31] we only consider the dominant contribution from gluon-gluon initiated transition, which proceeds through the process:

$g+g \rightarrow \sum_{f=1}^{N_{f}}\left(q_{R}^{f}+\bar{q}_{L}^{f}\right)+n_{g} g$

In the pseudo-particle picture, this can be interpreted as a decay process of a Instanton pseudo-particle with a mass $m_{I}$. For low Instanton masses, e.g. in the $50 \mathrm{GeV}$ range, we expect therefore an isotropic decay into up to 5 quarks, 5 anti-quarks as well as 5-10 gluons. The number of gluons is assumed to be Poisson distributed around an average, $\left\langle n_{g}\right\rangle$, which has been calculated in [31], and which in turn depends on $m_{I}$ and varies between 5 and 13 over the mass range $10 \mathrm{GeV}<m_{I}<$ $4 \mathrm{TeV}$. As a consequence, QCD Instanton-induced scattering processes produce soft bombs - a high-multiplicity spherically symmetric distributions of relatively soft particles [40]. These generic properties of QCD Instanton decays can be used to design experimental searches for these processes. The Instanton production cross section, reported in Table 1, falls rapidly with increasing mass of the Instanton pseudoparticle, $m_{I}$, or equivalently with the center of mass energy at parton level, $\sqrt{s^{\prime}}$ of the Instanton process. The cross section dependence on $\sqrt{s^{\prime}}$ is different from that of other SM processes. Experimentally, $m_{I}$ can be approximated by the 4-vector sum of all reconstructed charged particles with a certain minimal transverse momentum.

Depending on the range of $m_{I}$ considered, different regimes can be identified with different processes contributing as background and different signal to background ratios. While the decay of low mass Instantons, e.g. $m_{I}=30 \mathrm{GeV}$, results in events with a high multiplicity of low energetic charged particle tracks, for masses larger than $200 \mathrm{GeV}$ jets of hadronic particles are produced. In the low mass regime, soft$Q C D$ events originating from inelastic, non-diffractive processes constitute the dominant background due to their large production cross section. For higher Instanton masses, high$p_{\mathrm{T}}$ jet production processes ( $\mathrm{ard} Q C D$ ), as well as vectorboson and top anti-top quark pair production in the hadronic decay channels contribute.

\section{MC samples, detector simulation and experimental observables}

In the following section we describe the event generation and detector simulation configuration used for the signal and background sample, summarised in Table 2, as well as the experimental observables investigated.

For all generated samples a typical detector response has been simulated through the DELPHES framework [41] with settings corresponding to the ATLAS experiment, and without considering additional pile-up interactions. Pile-up activity could become a non-negligible source of background when selecting events with high multiplicity final states, and will require dedicated studies in LHC analyses that cannot be performed with parametrised simulations.

\subsection{Soft QCD processes}

Soft and diffractive QCD events constitute the largest part of the hadronic cross-section when no high $p_{\mathrm{T}}$ object is identified. Due to their non-perturbative nature, soft $Q C D$ processes are described by phenomenological models, typically based on a multiparton interaction (MPI) description, which have been tuned to data using a wide variety of reference measurements. Within this study, the soft $Q C D$ processes in the PYTHIA8 [42] generator with the Monash tune [43] and the 
Table 1 Overview of expected cross sections of Instanton processes with different values of $\sqrt{s_{\min }^{\prime}}[31]$

\begin{tabular}{lllllll}
\hline$\sqrt{s_{\text {min }}^{\prime}}(\mathrm{GeV})$ & 10 & 20 & 50 & 100 & 200 & 500 \\
\hline$\sigma(p p \rightarrow I \rightarrow X)(\mathrm{pb})$ & $1.7 \times 10^{11}$ & $6.3 \times 10^{9}$ & $4.1 \times 10^{7}$ & $8.0 \times 10^{4}$ & $1.1 \times 10^{2}$ & $3.5 \times 10^{-3}$ \\
\hline
\end{tabular}

Table 2 Overview of the MC samples used to model the Instanton signal process and the SM background processes

\begin{tabular}{|c|c|c|c|}
\hline Process & Generator & Main generator setting & \# Events \\
\hline QCD-Instanton (low-mass regime) & SHERPA & Instanton_MIN_MASS: 25. & 10,000 \\
\hline QCD-Instanton (low-mass regime) & SHERPA & Instanton_MIN_MASS: 50 . & 10,000 \\
\hline QCD-Instanton (medium-mass regime) & SHERPA & Instanton_MIN_MASS: 100 . & 10,000 \\
\hline QCD-Instanton (medium-mass regime) & SHERPA & Instanton_MIN_MASS: 200. & 10,000 \\
\hline QCD-Instanton (high-mass regime) & SHERPA & Instanton_MIN_MASS: 300 . & 10,000 \\
\hline QCD-Instanton (high-mass regime) & SHERPA & Instanton_MIN_MASS: 500 . & 10,000 \\
\hline QCD-Instanton (high-mass regime) & SHERPA & Instanton_MIN_MASS: 1000. & 10,000 \\
\hline softQCD & PYTHIA8 & SOFTQCD:ALL = ON - Monash Tune & $1,000,000$ \\
\hline softQCD & PYTHIA8 & SOFTQCD:ALL $=$ ON - A14 Tune & $1,000,000$ \\
\hline softQCD & SHERPA & & $1,000,000$ \\
\hline softQCD & HERWIG & & $1,000,000$ \\
\hline $\begin{array}{l}q q \rightarrow X, q g \rightarrow X, g g \rightarrow X \\
(\text { hardQCD) }\end{array}$ & PYTHIA8 & $\begin{array}{l}\text { HARDQCD }: \text { ALL }=\text { ON } \\
\text { PHASESPACE:PTHATMIN }=5 .\end{array}$ & $1,000,000$ \\
\hline $\begin{array}{l}q q \rightarrow X, q g \rightarrow X, g g \rightarrow X \\
(\operatorname{hard} Q C D)\end{array}$ & PYTHIA8 & $\begin{array}{l}\text { HARDQCDALL }=\text { ON } \\
\text { PHASESPACE }: \text { PTHATMIN }=100 .\end{array}$ & $1,000,000$ \\
\hline $\begin{array}{l}q q \rightarrow X, q g \rightarrow X, g g \rightarrow X \\
(\text { hardQCD) }\end{array}$ & PYTHIA8 & $\begin{array}{l}\text { HARDQCDALL }=\text { ON } \\
\text { PHASESPACE: } \text { PTHATMIN }=300 .\end{array}$ & $1,000,000$ \\
\hline$W \rightarrow q \bar{q}+X$ & PYTHIA8 & WEAKSINGLEBOSON:FFBAR2W $=$ ON & $1,000,000$ \\
\hline$Z \rightarrow q \bar{q}+X$ & PYTHIA8 & WEAKSINGLEBOSON:FFBAR2W $=$ ON & $1,000,000$ \\
\hline$W W \rightarrow q \bar{q} q \bar{q}+X$ & PYTHIA8 & & $1,000,000$ \\
\hline$t \bar{t} \rightarrow b q \bar{q}+\bar{b} q \bar{q}+X$ & PYTHIA8 & TOP:ALL $=$ ON & $1,000,000$ \\
\hline
\end{tabular}

NNPDF23LO PDF set [44] are used as the baseline. In total, one million events have been generated for proton-proton collisions at a center of mass energy of $13 \mathrm{TeV}$.

We also consider softQCD production in PYTHIA8 using a different shower and multi-parton interaction tune, A14 [45], as well as the predictions of soft $Q C D$ processes from the HERWIG7 [46] and the SHERPA [47,48] event generators. We compare normalised distributions for a number of observables (defined in Sec. 3.4) of interest for Instanton processes in Fig. 4 for events with a reconstructed invariant mass based on reconstructed tracks between $20 \mathrm{GeV}$ and $40 \mathrm{GeV}$. A good agreement is observed between the different predictions for most distributions, with the possible exception of the $N_{\text {Displaced }}$ distribution, where the SHERPA predictions differs by about $20 \%$ from the other generators. In the following, he maximal difference between the various soft $Q C D$ samples is taken as systematic uncertainty on the nominal soft $Q C D$ prediction taken from PYTHIA8.

\subsection{Hard QCD and other processes}

High- $p_{\mathrm{T}}$ jet production processes can be predicted with high accuracy in perturbation theory, with inclusive jet production known up to NNLO []. We use PYTHIA8 with the NNPDF23LO PDF set to simulate LO hardQCD di-jet production, with additional jets included through additional parton scatterings. The transition between soft $Q C D$ and hardQCD processes is ambiguous and not well defined. In our study, we use the softQCD samples for all events, which have no jet at particle level with a transverse momentum above $20 \mathrm{GeV}$, while the simulation of hardQCD processes is used for all other events.

In addition to multi-jet final states, the production of topquark pairs and of $W$ and $Z$ bosons and di-boson processes can also lead to final states with high multiplicity of particles, in particular in their fully hadronic decay channels. These processes are also simulated at LO with PYTHIA8 and the 

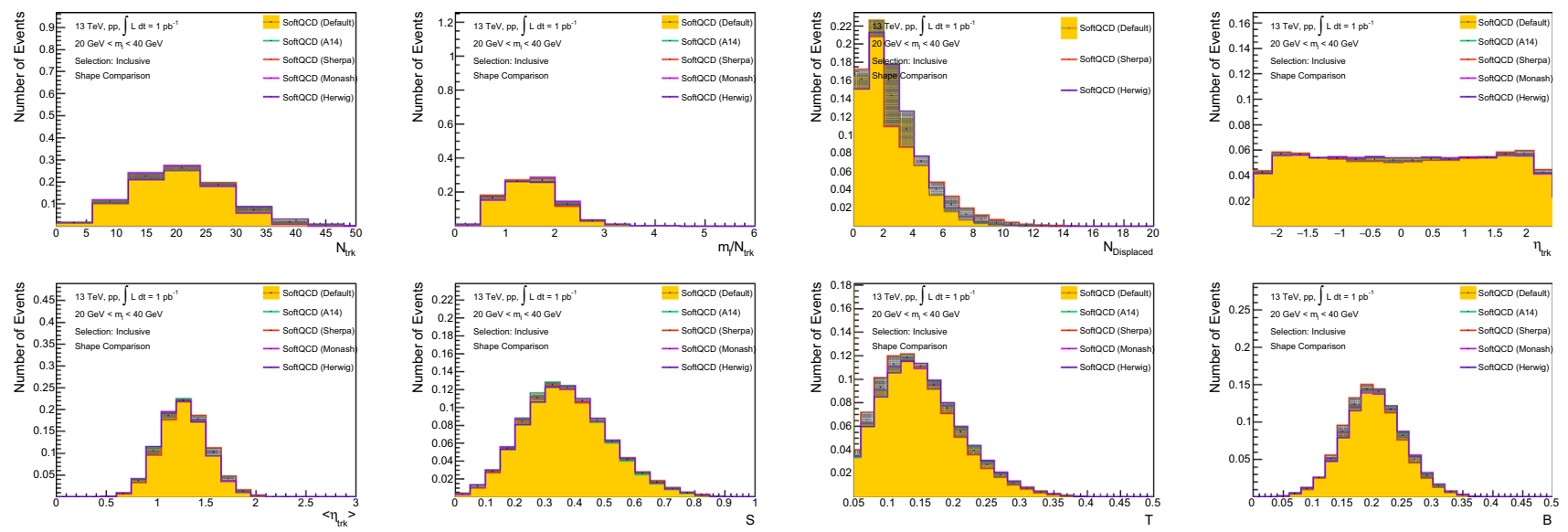

Fig. 4 Normalized distributions for softQCD processes with an invariant mass based on all tracks between $20 \mathrm{GeV}$ and $40 \mathrm{GeV}$, predicted be different MC generators or generator settings: $N_{\text {Trk }}, m_{I} / N_{\text {Trk }}, N_{\text {Displaced }}, \eta_{T r k},\left\langle\left|\eta_{T r k}\right|\right\rangle, \mathcal{S}, \mathcal{B}, \mathcal{T}$

NNPDF23LO PDF set. An uncertainty of $10 \%$ on all predictions of multi-jet, $t \bar{t}$ and vector boson processes are assumed in the following, to account for all theoretical uncertainties from missing higher perturbative orders and the modelling of effects from shower, hadronisation and MPI. However, it should be noted that it is possible to simulate these processes at higher perturbative accuracy. In addition, a realistic data analysis could use the leptonic decay channels of vectorboson and $t \bar{t}$ production to validate the theoretical predictions in dedicated control regions, thus reducing the model uncertainties.

\subsection{Signal simulation}

The Instanton signal samples have been produced with a modified version of the SHERPA event generator $[31,47,48]$. The predicted cross sections for Instanton production at different Instanton masses, $m_{I}$, have been implemented based on the calculations in [31] and are shown in Fig. 5. The figure also shows the predicted dependence of the Instanton cross section on the center of mass energy of proton-proton collisions for two different values of $\sqrt{s_{\min }^{\prime}}$, where also cross sections for soft- and hard-processes are shown in comparison. It is interesting to note that the Instanton cross sections exhibit a different dependence on $\sqrt{s}$ than soft $Q C D$ and hardQCD processes.

The decay of the Instanton pseudo-particle in the SHERPA implementation proceeds as follows [31]: firstly, the particle content of the final state is determined, where quark-antiquark pairs $q \bar{q}$, starting from the lowest mass, are added as long as the mass of the quark $m_{q}$ is smaller than a kinematics dependent threshold $\mu_{q}, m_{q}<\mu_{q}$ and as long as the combined mass of all pair-produced quarks is smaller than the Instanton mass. In a second step, the number of additional gluons is determined according to a Poissonian distribution with mean $\left\langle n_{g}\right\rangle$. The Rambo algorithm [49] is then used to distribute isotropically momenta to all decay products in the rest-frame of the Instanton pseudo-particle and boosted back to the lab-frame. The subsequent showering and hadronisation is based on the standard SHERPA implementation. The shapes of selected experimental observables for Instanton processes is shown in Fig. 6 for a mass range of $500 \mathrm{GeV}$ to $800 \mathrm{GeV}$. For comparison, the same distribution for the hardQCD processes with a minimal energy $s^{\prime}$ of $500 \mathrm{GeV}$ are overlaid.

To efficiently populate the full range of Instanton masses, a total of six Instanton signal samples have been produced, each covering an exclusive mass range between $20 \mathrm{GeV}$ and $600 \mathrm{GeV}$. An overview is given in Table 2 .

In order to validate the main properties of the Instanton decay in the SHERPA implementation, additional samples of the similar decay in the HERWIG7 generator [50] have been produced, where isotropic decays of pseudo-particles with masses between $500 \mathrm{GeV}$ and $800 \mathrm{GeV}$ into $2 \cdot N_{f}$-quarks and $n_{g}$ gluons have been simulated and reasonable agreement has been observed.

\subsection{Experimental observables}

Several observables sensitive to Instanton processes have been studied at detector level. The basic input quantities used are the four-vectors of charged particles reconstructed as particle tracks as well as particle jets, reconstructed using an anti$\mathrm{k}_{T}[51,52]$ algorithm with a radius parameter of 0.4 . Tracks are assumed to be massless and hence their four-vectors are defined by their transverse momentum, $p_{T}$, in the $x-y$ plane, ${ }^{1}$ the polar angle $\theta$ measured from the positive $z$ axis, as well as the azimuthal angle $\phi$ in the $x-y$ plane. The polar

\footnotetext{
${ }^{1}$ Transverse to the beam axis.
} 

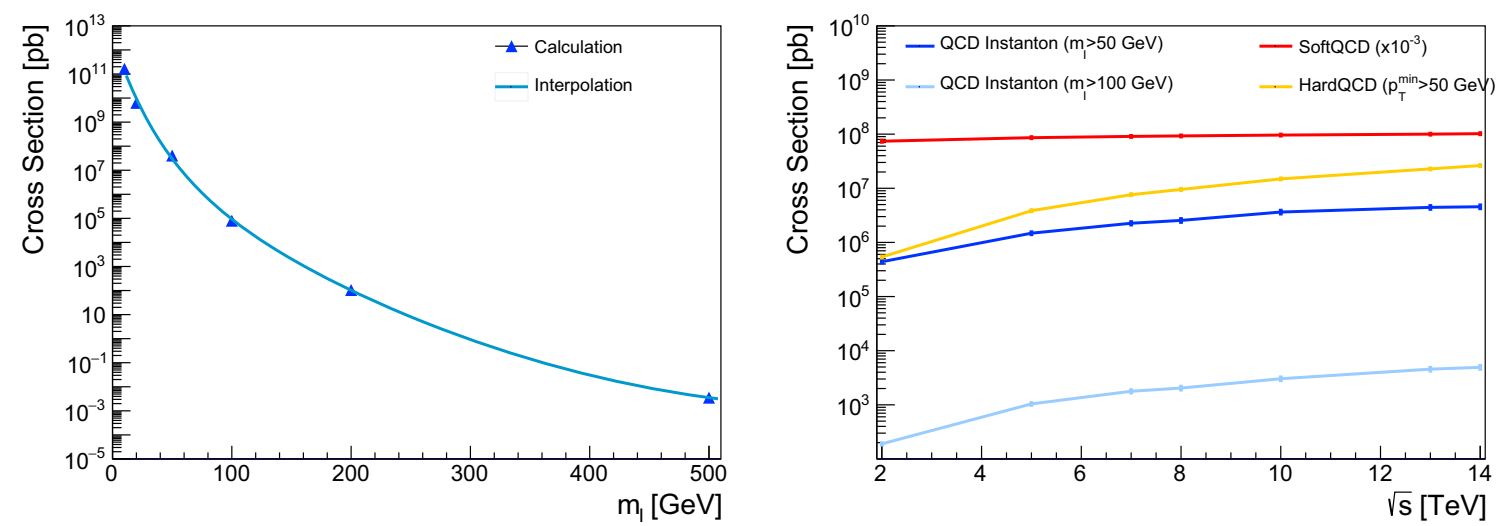

Fig. 5 The production cross section of Instanton processes in proton proton collisions at $\sqrt{s}=13 \mathrm{TeV}$ as a function of their mass (left) and a comparison of the cross section dependence on $\sqrt{s}$ between the Instanton signal and various background processes (right)
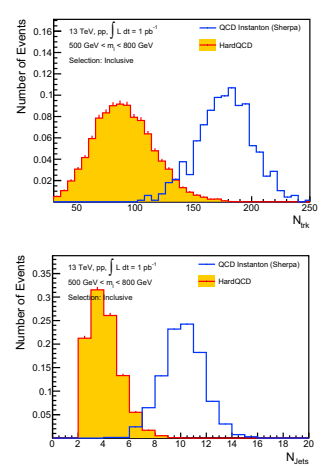
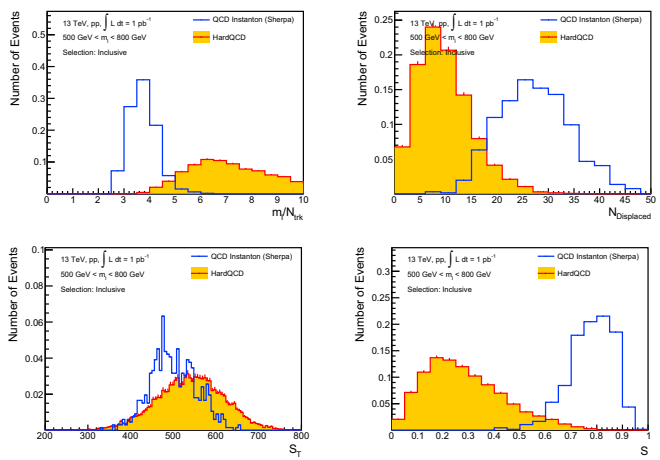
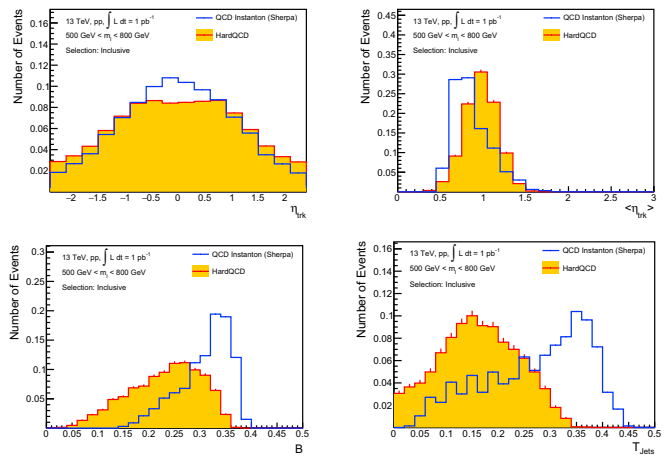

Fig. 6 Normalized distributions of the Instanton signal sample, generated by Sherpa with Instanton masses between $500 \mathrm{GeV}$ and $800 \mathrm{GeV}$ as well as for hardQCD processes in a similar mass range: $N_{\text {Trk }}, m_{I} / N_{\text {Trk }}, N_{\text {Displaced }}, \eta_{T r k},\left\langle\left|\eta_{T r k}\right|\right\rangle, N_{\mathrm{Jets}}, S_{T}^{\text {tracks }}, \mathcal{S}, \mathcal{B}, \mathcal{T}$

angle is expressed in terms of the pseudorapidity $\eta$, defined by $\eta=-\ln (\tan \theta / 2)$. We require transverse momenta greater than $500 \mathrm{MeV}$ for reconstructed tracks, as well as a maximal absolute pseudo-rapidity of 2.5. Particle jets are required to have at least a transverse momentum of $20 \mathrm{GeV}$ and $\eta<2.5$.

A first experimental observable for the selection of Instanton processes is the number of reconstructed tracks, $N_{\text {Trk }}$, as well as the number of reconstructed jets $N_{\text {jet }}$. Since many charged decay particles are expected from the decay of an Instanton pseudo-particle with a given mass, the ratio between the mass and the number of tracks, $m_{I} / N_{\text {Trk }}$ is also of interest. Similarly, the scalar sum of the transverse momenta $p_{\mathrm{T}}$ of all charged tracks (or particle-jets), $S_{T}=\sum\left|p_{\mathrm{T}}^{i}\right|$ in dependence of $m_{I}$ is studied. Isotropic decays of resonances are expected to have more central than forward activity, i.e. the pseudo-rapidity distribution of all charged tracks, $\eta_{T r k}$, as well as the average pseudo-rapidity of charged tracks per event $\left\langle\eta_{T r k}\right\rangle$ are expected to be sensitive. Due to the presence of $c$ - and $b$ - quarks as decay products of the Instanton and the relatively long life-times of the corresponding hadronised mesons, one might expect a higher number of charged particles displaced vertices compared to other SM processes. The number of reconstructed charged particles tracks with a production vertex that has a distance in the transverse plane of more than $0.02 \mathrm{~mm}$ to the primary vertex of the collision, $N_{\text {Displaced }}$, is therefore also studied.

We also consider variables which are directly related to the expected isotropy of Instanton decays. One such variable is the event-sphericity $\mathcal{S}$, defined via the tensor $S$,

$S^{\alpha \beta}=\frac{\sum_{i} p_{i}^{\alpha} p_{i}^{\beta}}{\sum_{i}\left|\vec{p}_{i}\right|^{2}}$,

where the indices denote the $x, y$, and $z$ components of the momentum of the particle $i$ in its rest-frame. The sphericity of the event is constructed using the two smallest eigenvalues of this tensor, $\lambda_{2}$ and $\lambda_{3}$, i.e. $\mathcal{S}=\frac{3}{2}\left(\lambda_{2}+\lambda_{3}\right)$ and takes values between 0 and 1 . A fully balanced dijet events leads to a sphericity of $\mathcal{S}=0$, while a fully isotropic event has a sphericity of $\mathcal{S}=1$. A similar event shape variable is the thrust $\mathcal{T}$, defined as

$\mathcal{T}=1-\max _{\vec{n}} \frac{\sum_{i}\left|\vec{p}_{i} \cdot \vec{n}\right|}{\sum_{i}\left|\vec{p}_{i}\right|}$,

where $\vec{n}$ is a unit vector. Fully spherical symmetric events yield $\mathcal{T}=0.5$, while fully balanced dijet events have $\mathcal{T}=0$. The definition of thrust also defines the thrust axis $\vec{n}$, which 
maximises the value of $\mathcal{T}$. The thrust axis defines a left $\mathcal{L}$ and right $\mathcal{R}$ hemisphere for each event, which can be used to define the jet broadening of an event. The left and right broadening is defined as

$\mathcal{B}_{\mathcal{L}}=\sum_{i \in \mathcal{L}} \frac{\left|\vec{p}_{i} \times \vec{n}\right|}{\sum_{i}\left|\vec{p}_{i}\right|}$ and $\mathcal{B}_{\mathcal{R}}=\sum_{i \in \mathcal{R}} \frac{\left|\vec{p}_{i} \times \vec{n}\right|}{\sum_{i}\left|\vec{p}_{i}\right|}$

The total jet broadening $\mathcal{B}$ is then defined as $\mathcal{B}=\mathcal{B}_{\mathcal{L}}+$ $\mathcal{B}_{\mathcal{R}}$, and behaves similar as $\mathcal{T}$, i.e. is 0 and 0.5 for dijet and spherically symmetric events, respectively.

The sphericity $\mathcal{S}$, the thrust $\mathcal{T}$ as well as the total jet broadening $\mathcal{B}$ are calculated twice, using all reconstructed tracks and using all reconstructed jets in an event. The calculation is based on the code provided in [53]. It should be noted that these three observables are significantly correlated. An additional correlation is observed between $\mathcal{S}$ and the $\eta_{T r k}$ distribution as events with large values of $\mathcal{S}$ tend to enhance the number of tracks in the central region, i.e. with $|\eta|<1.0$.

\section{Search strategies}

In contrast to most searches for new particles, no resonance behaviour is expected for Instanton induced processes, but rather a continuous, rapidly falling excess in the spectrum of invariant mass of all hadronic final state objects. This provides significant challenges in the search for Instanton-induced processes. While sizeable cross sections are expected for small Instanton masses, the experimental signatures in this energy range might be difficult to distinguish from soft QCD activity. At high luminosities, the large amount of expected pile-up events would further complicates such a search. In the high energy regime, the experimental signatures of Instanton-induced processes would be striking, their cross sections are however highly suppressed and hence difficult to observe in the first place.

The expected invariant mass distribution of reconstructed tracks is shown in Fig. 7 for the Instanton processes and the SM backgrounds, scaled to the expected event yields for an integrated luminosity of $\int L d t=1 \mathrm{pb}^{-1}$. At low invariant masses, the softQCD processes are the dominating background, while for high invariant masses hardQCD processes as well as top-quark pair and electroweak boson production become relevant. The signal over background ratio falls rapidly with increasing mass, suggesting a higher chance of observing of Instanton processes in the low mass regime. As expected, the high multiplicity final state for the Instanton processes make the number of reconstructed charged particle tracks and the number of reconstructed jets in the event powerful discriminants against background. Figure 7 shows the invariant mass distribution for signal and background processes when requiring at least eight reconstructed jets with a minimal momentum of $20 \mathrm{GeV}$. The expected signal to background ratio increases by several orders of magnitude, remaining below $10^{-4}$ with the expectation of 1 Instanton event for an integrated luminosity of $\int L d t \approx 0.1 \mathrm{fb}^{-1}$. This highlights the challenge of observing of Instanton processes for masses of several hundred $\mathrm{GeV}$.

In the following, we will present and discuss possible analysis strategies. To this aim, we found useful to distinguish four different mass ranges: $20<\sqrt{s^{\prime}}$ min $<40 \mathrm{GeV}$ and $40<\sqrt{s^{\prime}}$ min $<80 \mathrm{GeV}$ for the low mass regime, where soft$Q C D$ processes dominate, $200<\sqrt{s^{\prime}}$ min $<300 \mathrm{GeV}$ for the medium mass regime where hardQCD processes dominate and $300<\sqrt{s^{\prime}}$ min $<500 \mathrm{GeV}$ for the high mass regime, where also top-quark pair productions becomes relevant.

Different signal selections have been studies, optimised on the signal to background ratio. In addition, at least two control regions are defined for each mass range. These regions are designed to have only a small signal contribution and could therefore be used for the validation of the modelling of background processes. In a real analysis, the control regions can be used for an ABCD-based background estimation technique, i.e. to determine the background contribution in the signal regions in a fully data-driven way.

\subsection{Very low instanton masses: the soft QCD regime}

The very low Instanton mass regime is defined for two regions: the first requires the invariant mass of reconstructed tracks, $m_{I}$, between 20 and $40 \mathrm{GeV}$, the second between 40 and $80 \mathrm{GeV}$. The average $m_{I}$ values for Instantons in both regions are $24 \mathrm{GeV}$ and $46 \mathrm{GeV}$, respectively. A veto on $20 \mathrm{GeV}$ jets at reconstruction level is applied for both mass ranges. This requirement is applied to keep these regions orthogonal to the regions where hard $Q C D$ processes dominate. $^{2}$

The lower mass region is discussed first: Fig. 8 shows the predicted distributions of the event sphericity, $\mathcal{S}$ and the pseudo rapidity, $\eta_{T r k}$, of reconstructed charged particles for the various processes considered. The distributions are scaled to the expected event yields for an integrated luminosity of $L=\int 1 \mathrm{pb}^{-1}$. While soft $Q C D$ processes dominate the background, the Instanton signal is enhanced at large values of the sphericity and predicts a more central $\eta$ tracks distribution.

It is illustrative to compare the signal and background shapes for various observables. An overview of eight relevant observables, previously introduced, is shown in Fig. 9. Instantons processes are expected to have larger track multiplicities and hence smaller values of $m_{I} / N_{\text {Trk }}$. As expected, the observables related to the event topology indicate more

\footnotetext{
$\overline{2}$ Only hardQCD events with at least one jet on particle level with $p_{\mathrm{T}}>$ $20 \mathrm{GeV}$, that has either not been reconstructed or reconstructed with a smaller value of $p_{\mathrm{T}}$, pass the signal selection
} 


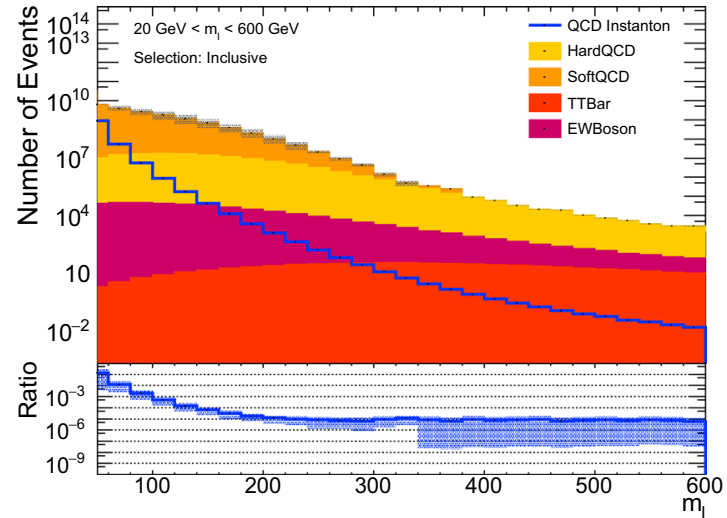

Fig. 7 Invariant mass distribution of all reconstructed tracks for standard model background processes and Instanton processes (left) as well as the same distribution for events with at least ten reconstructed particle jets with a $p_{\mathrm{T}}>20 \mathrm{GeV}$ (right). The events correspond to an integrated

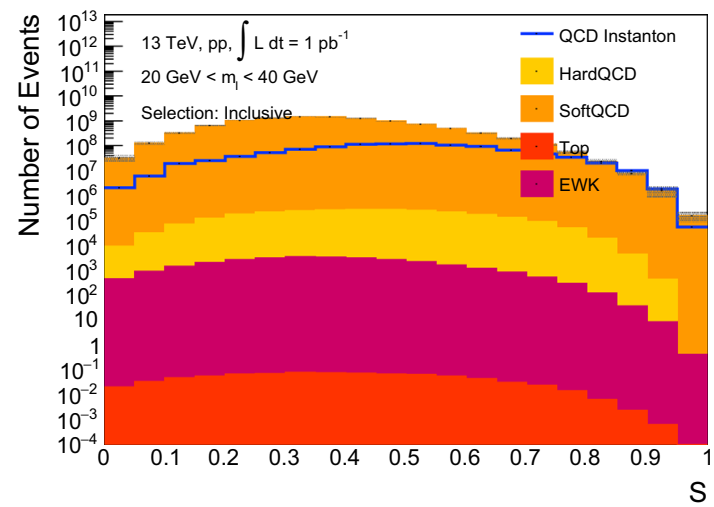

Fig. 8 Predicted distributions of the event sphericity, $\mathcal{S}$ (left), and pseudo rapidity, $\eta$, of reconstructed charged particles (right) for various processes, normalised to the expected event yields for an integrated luminosity of $L=\int 1 \mathrm{pb}^{-1}$. The invariant mass of all reconstructed

spherical events compared to the background processes. Highly interesting is the distribution of $N_{\text {Displaced }}$, i.e. the number of tracks with a displaced origin, as it differs significantly for soft- and hard $Q C D$ processes and the signal process. This behaviour might be explained by the fact that more heavy quarks in the final state of the Instanton decays are expected, which typically hadronise to long(er) lived mesons and baryons. Based on these distributions, a few signal selection scenarios have been developed and are summarised in Table 3. No requirements are made specifically for the event sphericity as well as the pseudo-rapidity of tracks. The idea behind this approach is, that these distributions could then be used as final discriminating variables in a combined fit of signal and background templates to data in order to extract a limit on the Instanton signal-strength.

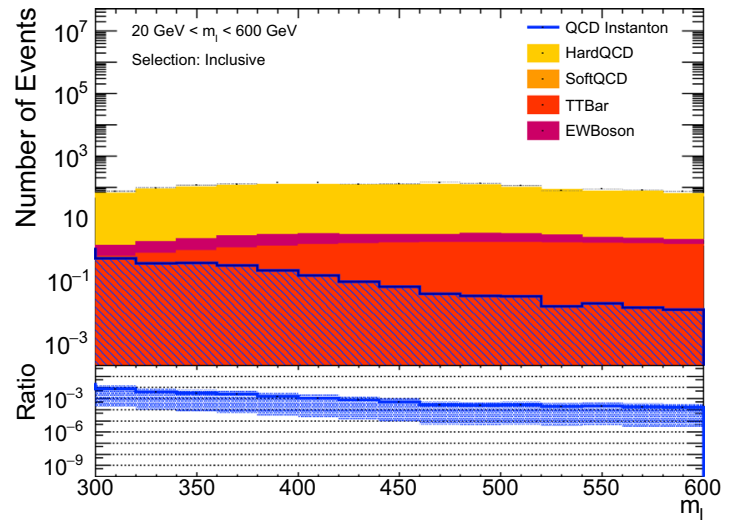

luminosity of $L=\int 1 \mathrm{pb}^{-1}$ and the distributions from all processes except the Instanton process are stacked. The model uncertainties are indicated as bands. The lower plots show the signal over background ratio corresponding to the upper row

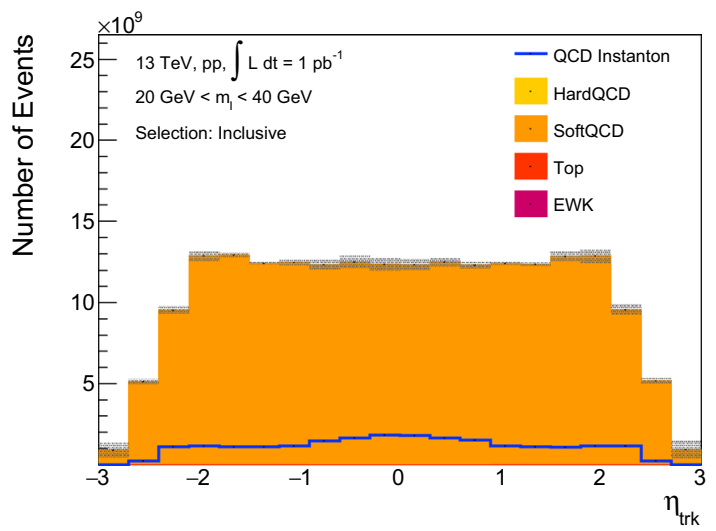

tracks is required to be between 20 and $40 \mathrm{GeV}$ (very low mass regime). The distributions from all SM processes except for the Instanton are stacked. The model uncertainties are indicated as bands

The standard signal selection applies requirements on the $N_{\text {Trk }}, m_{I} / N_{\text {Trk }}$ and $N_{\text {Jets }}$ distributions, where the latter is required to be 0 to reject hard $Q C D$ processes. The resulting sphericity and track $\eta$ distributions for the signal and background processes is shown in Fig. 10. An improvement by a factor of two in the signal over background ratio becomes visible after these selections are applied. In particular, the expected number of Instanton events becomes larger than the total SM background for event with sphericities $\mathcal{S}>0.85$, which is used to define the signal region selections in the following. The event-shape signal selection adds additional requirements on $\mathcal{B}$ and $\mathcal{T}$, hence affecting also the $\mathcal{S}$ distribution. The observables after this selection are shown in Fig. 11. The signal over background ratio improves further when also a requirement of $N_{\text {Displaced }}>6$ is applied, which 

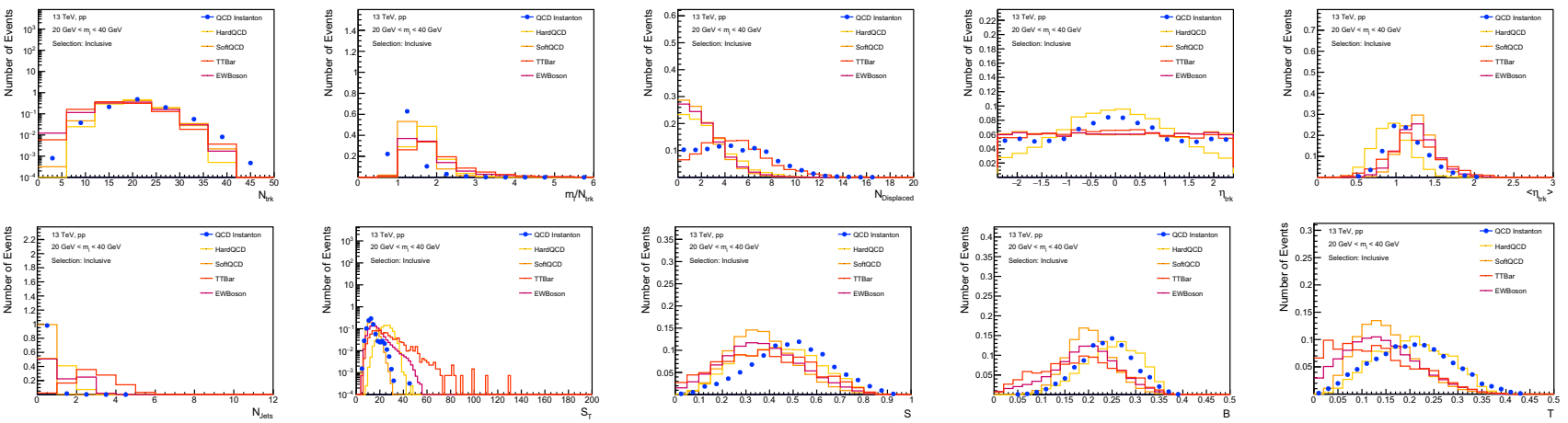

Fig. 9 Normalized distributions in the very low mass regime for various processes: $N_{\text {Trk }}, m_{I} / N_{\text {Trk }}, N_{\text {Displaced }}, \eta_{T r k},\left\langle\left|\eta_{T r k}\right|\right\rangle, N_{\text {Jets }}, S_{T}^{\text {tracks }}, \mathcal{S}, \mathcal{B}$, $\mathcal{T}$

defines our tight signal selection (Table 3). The resulting sphericity distribution for the tight signal selection is shown in Fig. 11. A very clean Instanton signal is expected for this tight selection.

Two possible definitions of control regions, called A and B, are summarised in Table 3. Both exhibit a signal contamination smaller than $10 \%$. The $N_{\text {Displaced }}$ distribution for control region $\mathrm{A}$, as well as the sphericity distribution for control region B, are shown in Fig. 12.

The calculation of Instanton cross sections relies on a semiclassical approximation which breaks down when the coupling is very large. This effect is exemplified by the fact that the Instanton cross section rises beyond the total proton-proton cross section for very low values of $s_{m} i n$. We thus also study a higher range of Instanton masses, $40<m_{I}<80 \mathrm{GeV}$, where we expect the cross section predictions for Instanton processes should be more reliable. In this regime, the softQCD background is still the dominant one in most regions of the phase space. Analogously to the previous case, three different signal selection scenarios and two control regions are defined, which is summarised in Table 4

The sphericity distribution for the signal and background processes for an inclusive selection ${ }^{3}$ in this mass range, as well as the three signal region definitions (standard, event-shape, tight) are shown in Fig. 13, together with the expected signal and background events in the signal region in Table 4. The standard and event-shape selections yield signal to background ratios below 1 and are dominated by soft$Q C D$ processes. The tight selection significantly enhances the signal and would allow for a clear observation. In this selection the dominant background contribution in the signal region comes from hardQCD processes. We note however that the negligible contribution from softQCD could just be a consequence of the limited statistics employed in this study, and it is possible that the actual background

\footnotetext{
3 Inclusive is defined here as only applying selection cuts on the reconstructed invariant mass $m_{I}$ of the Instanton
}

from soft $Q C D$ processes is larger.The interplay between soft$Q C D$ and hardQCD processes could be experimentally studied, by applying various requirements on the number of reconstructed jets and thus define different control regions.

\subsection{Medium instanton masses: the hard QCD regime}

Increasing the rangne of Instanton masses considered, one enters the regime of perturbative QCD, and the background prediction becomes less uncertain. A medium mass range with $200<m_{I}<300 \mathrm{GeV}$ with an average Instanton mass of $220 \mathrm{GeV}$ was studied. The $S_{T}^{\text {tracks }}$ distribution and the number of reconstructed jets with $p_{\mathrm{T}}>20 \mathrm{GeV}$ in this mass range is shown in Fig. 14 for the signal and background processes. The Instanton processes are expected to peak for $3 \leq N_{j e t} \leq 6$, and one can see events with $S_{T}>150 \mathrm{GeV}$ have only a negligible contribution form softQCD processes.

A standard signal selection imposes requirements on $N_{\text {Trk }}, m_{I} / N_{\text {Trk }}$ and the $N_{\text {Jets }}$ distribution. The event-shape selection applies in addition a minimum requirement on $\mathcal{B}$ and $\mathcal{T}$. Similarly to the low mass scenarios, a requirement on $N_{\text {Displaced }}$ is made for the tight signal selection. All cuts for the signal selection as well as the definitions of the two control regions are summarized in Table 5. The event-shape selection yields 1 signal event and approximately 10 background events for an integrated luminosity of $\int L d t=1 \mathrm{pb}^{-1}$. For the tight selection 0.5 signal events and 0.6 background events are expected. An observation would therefore be possible with an integrated luminosity of about $\int L d t \sim 10 \mathrm{pb}^{-1}$

In this context the ability of the LHC experiments to efficiently trigger on these event topologies becomes relevant. For the studies of softQCD processes at the LHC, special triggers are used, which record collision events even with limited activity in the detector, i.e. are nearly free of any bias towards a certain physics signature. Given the enormous rates of such minimum bias triggers, only a small fraction of 
Table 3 Overview of the standard and tight signal selection as well as the definition of two control regions aiming at very low Instanton masses $\left(20 \mathrm{GeV}<m_{I}<40 \mathrm{GeV}\right)$

\begin{tabular}{|c|c|c|c|c|c|}
\hline & \multicolumn{3}{|c|}{$\underline{\text { Signal region }}$} & \multicolumn{2}{|c|}{ Control region } \\
\hline & Standard & Event-shape & Tight & A & $\mathrm{B}$ \\
\hline Invariant mass of rec. tracks (Instanton mass), $m_{I}$ & \multicolumn{5}{|c|}{$20 \mathrm{GeV}<m_{I}<40 \mathrm{GeV}$} \\
\hline \multicolumn{6}{|l|}{ Selection requirements } \\
\hline Number of rec. tracks, $N_{\text {Trk }}$ & $>20$ & $>20$ & $>20$ & $>15$ & $>20$ \\
\hline Number of rec. tracks/Instanton mass, $m_{I} / N_{\text {Trk }}$ & $<1.5$ & $<1.5$ & $<1.5$ & $>2.0$ & $<1.5$ \\
\hline Number of Jets, $N_{\text {Jets }}$ & $=0$ & $=0$ & $=0$ & $=0$ & $=0$ \\
\hline Broadening, $\mathcal{B}_{\text {Tracks }}$ & & $>0.3$ & $>0.3$ & $>0.3$ & $>0.3$ \\
\hline Thrust, $\mathcal{T}_{\text {Tracks }}$ & & $>0.3$ & $>0.3$ & $>0.3$ & $>0.3$ \\
\hline Number of displaced vertices, $N_{\text {Displaced }}$ & & & $>6$ & & $<4$ \\
\hline \multicolumn{6}{|c|}{ Expected events for $\int L d t=1 \mathrm{pb}^{-1}$ in the signal region $(\mathcal{S}>0.85)$} \\
\hline$N_{\text {Signal }}$ & $1.1 \times 10^{7}$ & $8.9 \times 10^{6}$ & $5.9 \times 10^{6}$ & $<1$ & $6.8 \times 10^{5}$ \\
\hline$N_{\text {Background }}$ & $6.2 \times 10^{6}$ & $4.3 \times 10^{6}$ & $1.8 \times 10^{5}$ & $3 \times 10^{5}$ & $3.3 \times 10^{6}$ \\
\hline
\end{tabular}

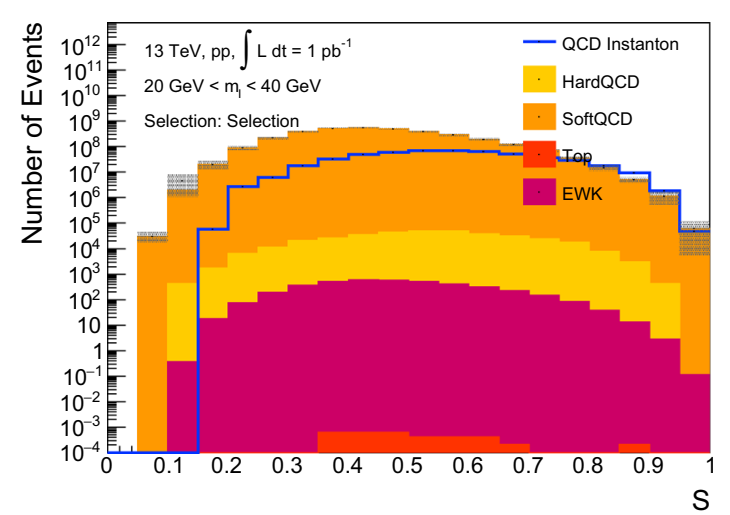

Fig. 10 Predicted distributions of the event sphericity (left) and pseudo rapidity, $\eta$, of reconstructed charged particles (right) for various processes, weighted by their predicted cross sections for an integrated luminosity of $L=\int 1 \mathrm{pb}^{-1}$ after the nominal selection. The invariant mass

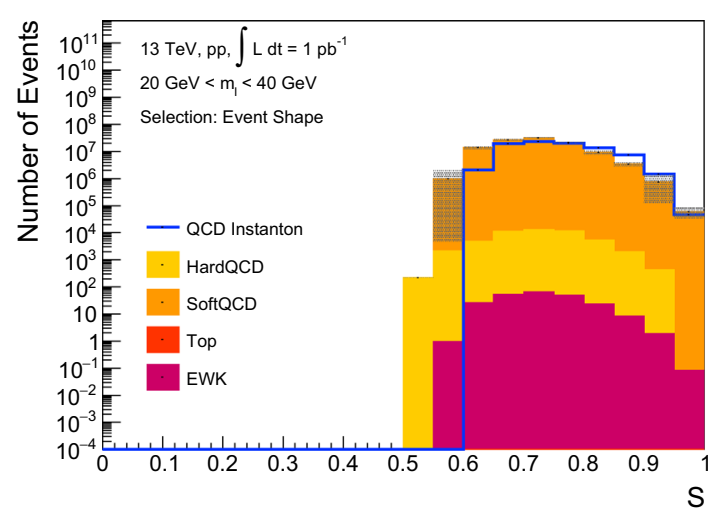

Fig. 11 Predicted distributions of the event sphericity for various processes, weighted by their predicted cross sections for an integrated luminosity of $L=\int 1 \mathrm{pb}^{-1}$ after the event-shape based selected (left) and tight selection (right). The invariant mass of all reconstructed tracks is

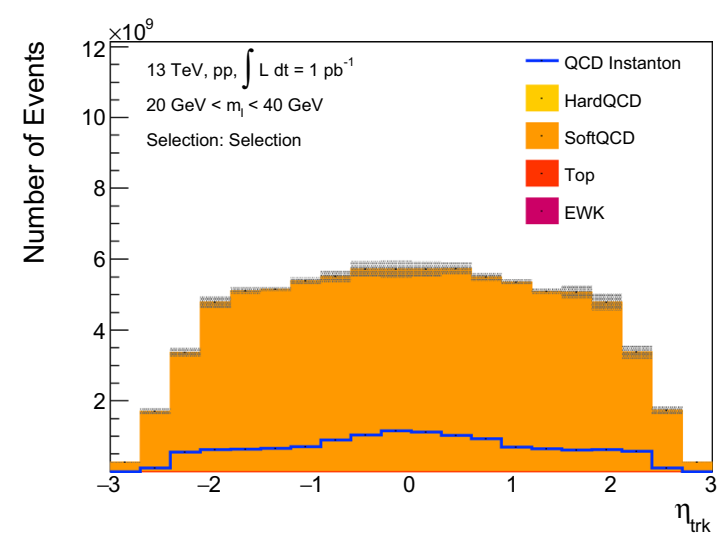

of all reconstructed tracks is required to be between $20 \mathrm{GeV}$ and $40 \mathrm{GeV}$ (very low mass regime). The distributions from all processes except the Instanton process are stacked. The model uncertainties are indicated as bands

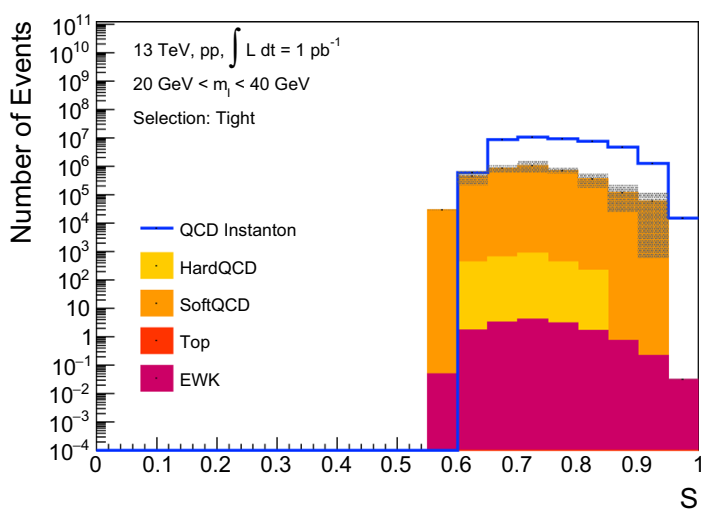

required to be between 20 and $40 \mathrm{GeV}$ (very low mass regime). The distributions from all processes except the Instanton process are stacked. The model uncertainties are indicated as bands 


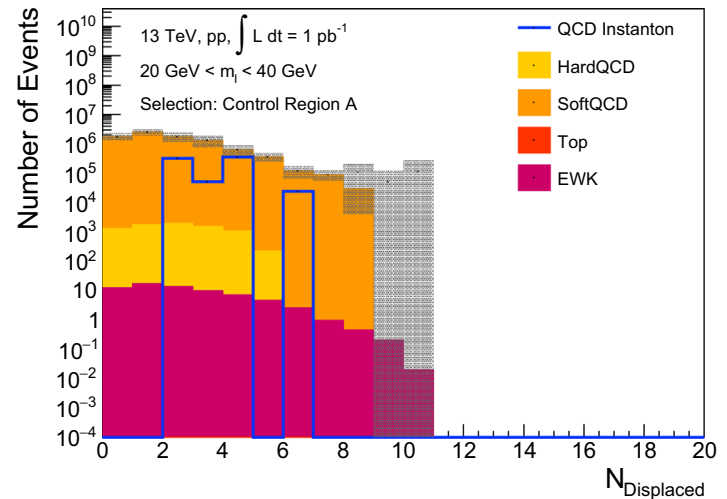

Fig. 12 Predicted distributions of the number of displaced tracks in the control region A (left) and the event sphericity in control region B (right), weighted by their predicted cross sections for an integrated luminosity of $L=\int 1 \mathrm{pb}^{-1}$. The invariant mass of all reconstructed tracks is

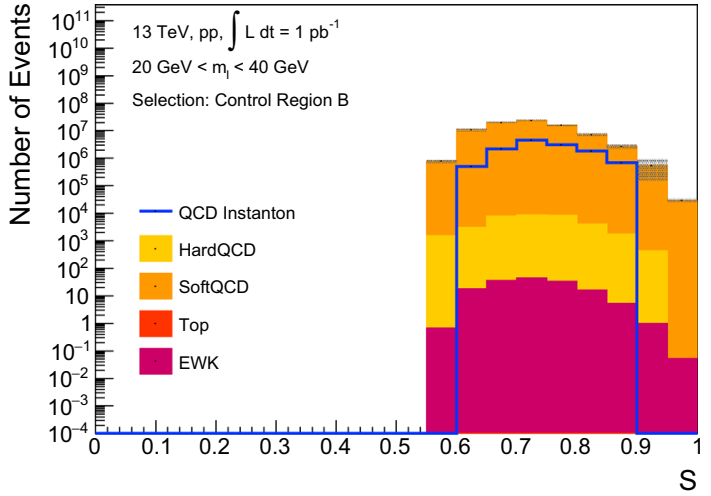

required to be between 20 and $40 \mathrm{GeV}$ (very low mass regime). The distributions from all processes except the Instanton process are stacked. The model uncertainties are indicated as bands

Table 4 Overview of the standard and tight signal selection as well as the definition of two control regions aiming at very low Instanton masses $\left(40 \mathrm{GeV}<m_{I}<80 \mathrm{GeV}\right)$

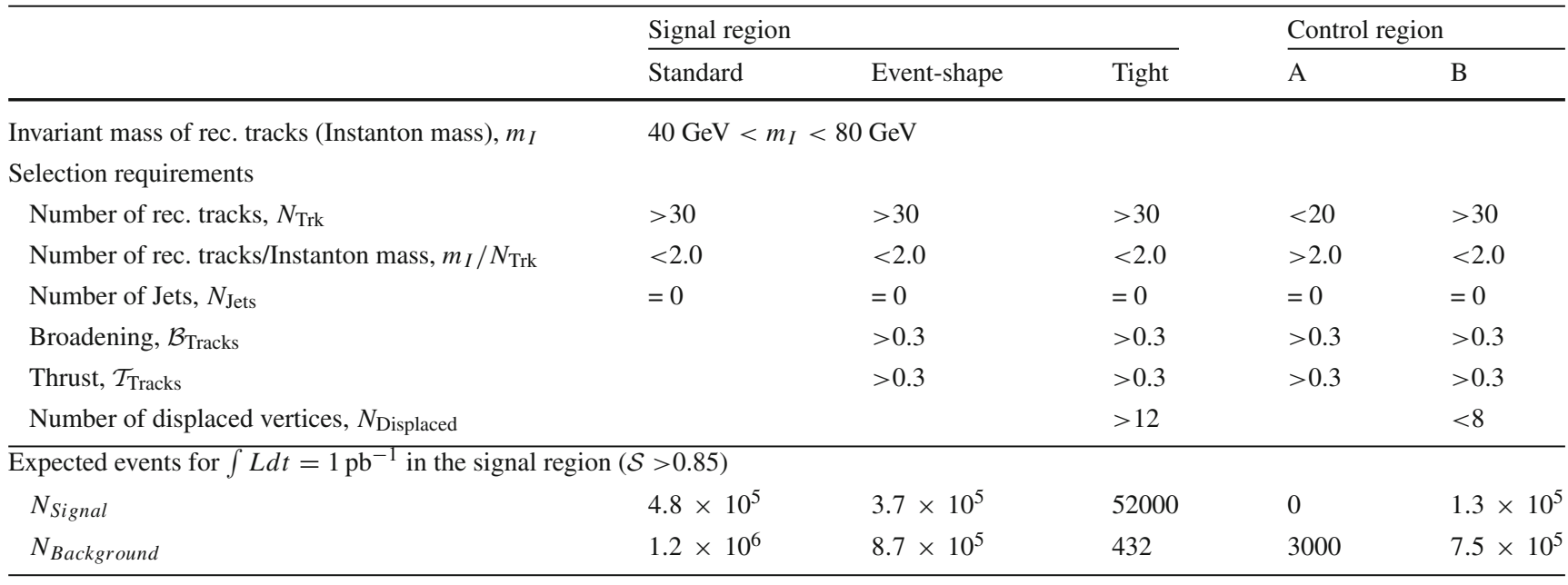

these events can be actually stored on tape and subsequently analysed. However, the published soft $Q C D$ analysis at the LHC indicate that sufficient statistics has been already collected to allow for Instanton searches in the low mass regime. This is not obviously the case for the medium and high Instanton mass regime, as the integrated luminosity required for an observation increases significantly. Typically, jet and multi-jet triggers require minimal transverse jet energies of $50 \mathrm{GeV}$ or more and hence are of no use. New trigger strategies might be needed the upcoming LHC runs to be able to record enough Instanton events, as pointed out independently in [32].

The predicted distributions of the event sphericity for the signal and background processes, scaled to the expected event yields for an integrated luminosity of $L=\int 1 \mathrm{pb}^{-1}$ is shown in Fig. 15 for the event-shape and the tight selection. The dominant background are multijet events from hard $Q C D$ processes. Figure 16 shows the predicted number of displaced tracks in the control region $\mathrm{A}$ as well as the event sphericity in control region $\mathrm{B}$, which again can be used to validate the modelling of the background processes.

\subsection{High instanton masses: the top quark regime}

The $300<m_{I}<500 \mathrm{GeV}$ mass range is also dominated by hardQCD multi-jet background. This can be seen in Fig. 14, which shows the event sphericity and the number of reconstructed particle jets with $p_{\mathrm{T}}>20 \mathrm{GeV}$ for signal and background events. However, it is interesting to apply a dedicated event selection that promotes another process as dominant background, not suffering from the same model uncertainties as the background in the medium mass regime. Hence we focus our selection here on top-quark pair events. While an obvious approach to enhance the top-quark (as well as the 

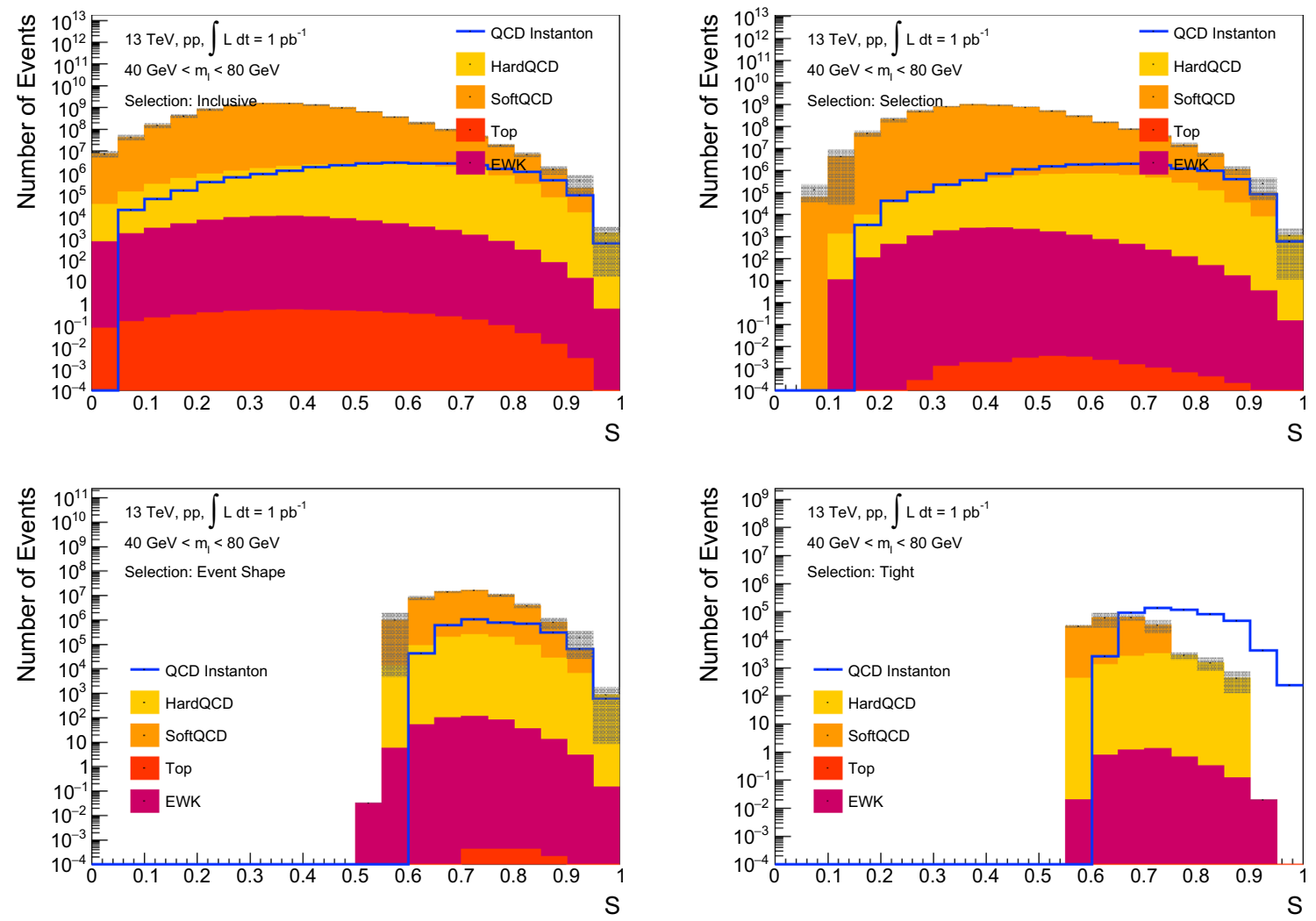

Fig. 13 Predicted distributions of the event sphericity for various processes, weighted by their predicted cross sections for an integrated luminosity of $L=\int 1 \mathrm{pb}^{-1}$ for an inclusive selection (upper left), the nominal selection (upper right), the event-shape based selection (lower left)

and the tight selection (lower right). The invariant mass of all reconstructed tracks is required to be between 40 and $80 \mathrm{GeV}$. The distributions from all processes except the Instanton process are stacked. The model uncertainties are indicated as bands
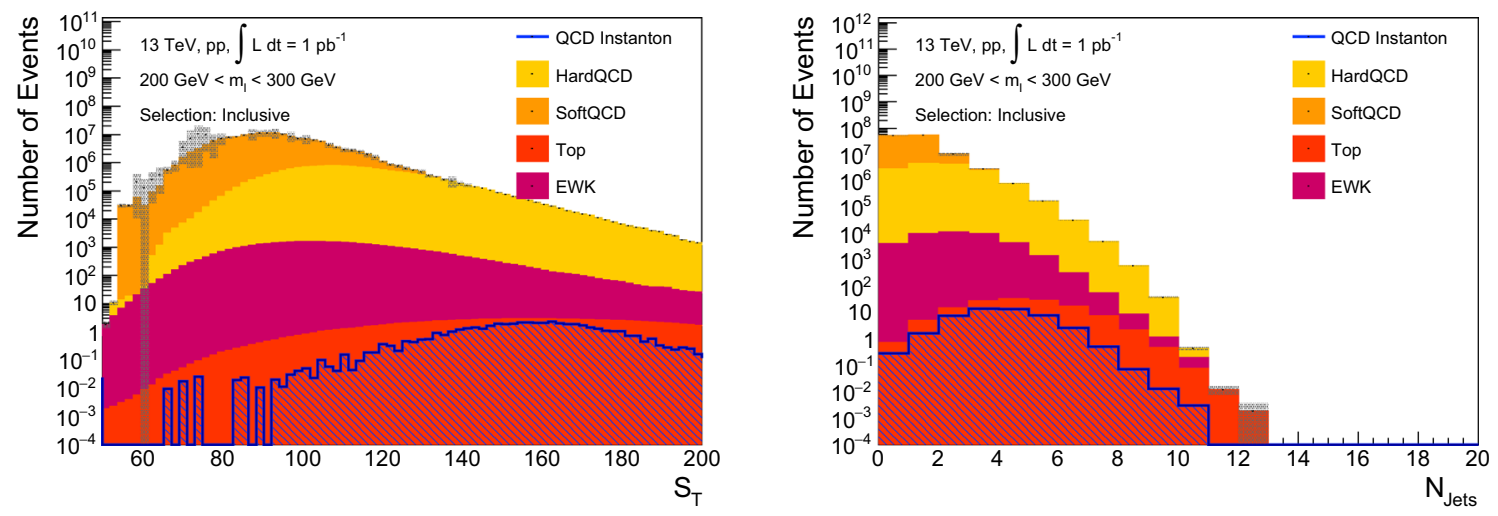

Fig. 14 Predicted distributions of the ST (left) and number of reconstructed jets (right) for various processes, weighted by their predicted cross sections for an integrated luminosity of $L=\int 1 \mathrm{pb}^{-1}$. The invariant mass of all reconstructed tracks is required to be between 200 and

$300 \mathrm{GeV}$ (medium mass regime). The distributions from all processes except the Instanton process are stacked. The model uncertainties are indicated as bands 
Table 5 Overview of the standard and tight signal selection as well as the definition of two control regions aiming at very low Instanton masses $\left(200 \mathrm{GeV}<m_{I}<300 \mathrm{GeV}\right)$

\begin{tabular}{|c|c|c|c|c|c|}
\hline & \multicolumn{3}{|c|}{ Signal region } & \multicolumn{2}{|c|}{ Control region } \\
\hline & Standard & Event-shape & Tight & A & B \\
\hline Invariant mass of rec. tracks (Instanton mass), $m_{I}$ & \multirow{2}{*}{\multicolumn{3}{|c|}{$200 \mathrm{GeV}<m_{I}<300 \mathrm{GeV}$}} & & \\
\hline \multicolumn{3}{|l|}{ Selection requirements } & & & \\
\hline Number of rec. tracks, $N_{\text {Trk }}$ & $>80$ & $>80$ & $>80$ & $>80$ & $>80$ \\
\hline Number of rec. tracks/Instanton mass, $m_{I} / N_{\text {Trk }}$ & $<3.0$ & $<3.0$ & $<3.0$ & $>3.0$ & $<3.0$ \\
\hline Number of Jets, $N_{\text {Jets }}$ & $3-6$ & $3-6$ & $3-6$ & $3-6$ & $3-6$ \\
\hline Broadening, $\mathcal{B}_{\text {Tracks }}$ & & $>0.3$ & $>0.3$ & $>0.3$ & $>0.3$ \\
\hline Thrust, $\mathcal{T}_{\text {Tracks }}$ & & $>0.3$ & $>0.3$ & $>0.3$ & $>0.3$ \\
\hline Number of displaced vertices, $N_{\text {Displaced }}$ & & & $>15$ & & $<10$ \\
\hline \multicolumn{6}{|l|}{ Results } \\
\hline \multicolumn{6}{|c|}{ Expected events for $\int L d t=1 \mathrm{pb}^{-1}$ in the signal region $(\mathcal{S}>0.85)$} \\
\hline$N_{\text {Signal }}$ & 5.6 & 1.0 & 0.54 & 0.04 & 0.21 \\
\hline$N_{\text {Background }}$ & 1900 & 9.6 & 0.64 & 200 & 1100 \\
\hline
\end{tabular}
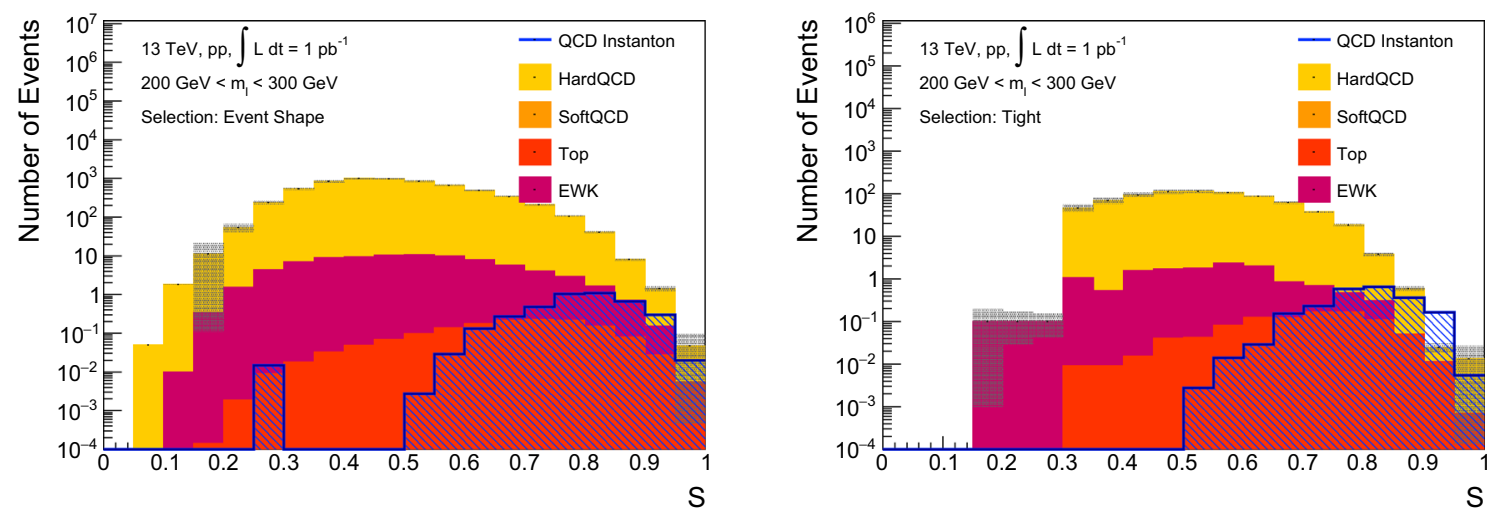

Fig. 15 Predicted distributions of $\mathcal{S}$ for an integrated luminosity of $L=\int 1 \mathrm{pb}^{-1}$ for the event-shape based selection (left) and the tight selection (right). The invariant mass of all reconstructed tracks is required to be between 200 and $300 \mathrm{GeV}$ (medium mass regime)

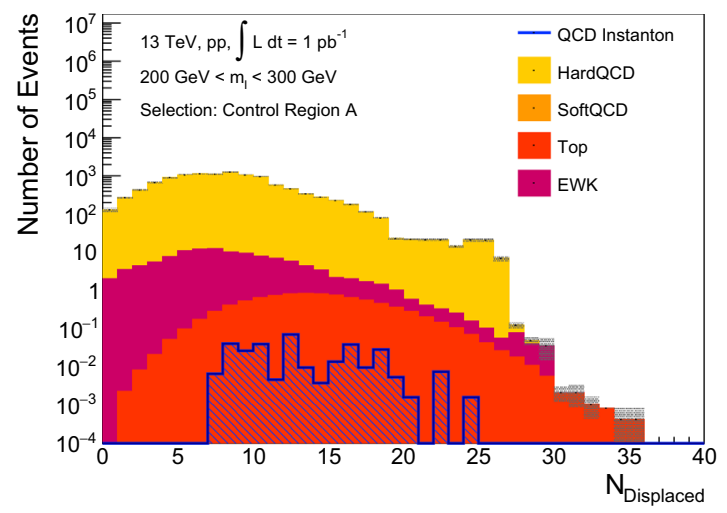

Fig. 16 Predicted distributions of the number of displaced tracks in the control region A (left) and the event sphericity in control region B (right), weighted by their predicted cross sections for an integrated luminosity of $L=\int 1 \mathrm{pb}^{-1}$. The invariant mass of all reconstructed tracks

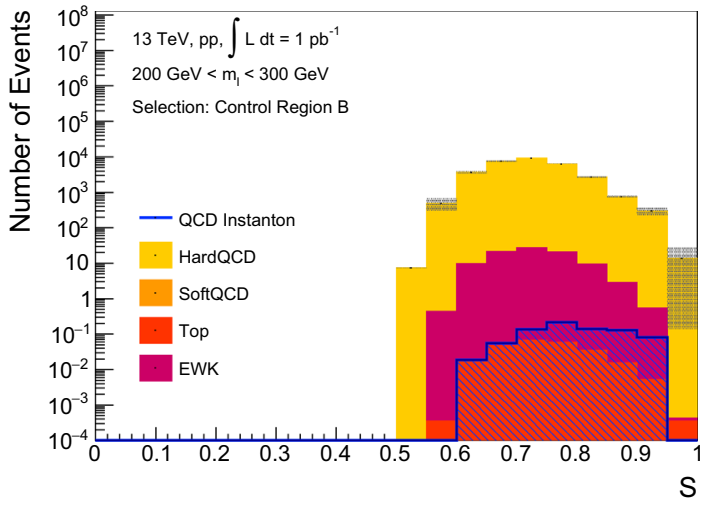

is required to be between 200 and $300 \mathrm{GeV}$ (medium mass regime). The distributions from all processes except the Instanton process are stacked. The model uncertainties are indicated as bands 
Instanton) contribution is to require reconstructed jets tagged to originate from $b$-hadrons, this was not required within this study due to the uncertainties in the signal simulation. Our results on the expected sensitivity are therefore conservative (Fig. 17).

The large track multiplicity in events within $300<m_{I}<$ $500 \mathrm{GeV}$ does not allow anymore for a clean separation between signal and background processes. Hence no signal selection cut involving $N_{\text {Trk }}$ is applied. The standard signal selection requires only $m_{I} / N_{\text {Trk }}<3.0$ as well as more than seven reconstructed jets with $p_{\mathrm{T}}>20 \mathrm{GeV}$. The latter requirement allowing to enhance the top-quark background contribution. The event-shape and tight signal selections follow the same lines as for the lower mass ranges, i.e. impose cuts on the event topology as well as on $N_{\text {Displaced. A sum- }}$ mary of all signal selection criteria is given in Table 6 . The corresponding event sphericity distributions for the eventshape and the tight selection is shown in Fig. 18, where the top-quark background starts to dominate large values of $\mathcal{S}$. written before b-tagging is not applied A further enhancement of the top-quark contribution can be achieved by an additional $b$-tagging requirement without impacting significantly the signal yield.

The advantage of this signal selection relies on the experimentally well understood top-quark pair production. In addition to similar control regions as in the low mass ranges, topquark enhanced regions can be envisioned, e.g. by requiring one additional reconstructed lepton (electron or muon) in the event, originating from the leptonic decay of one top-quark (see definition of Control Region $\mathrm{C}$ in Table 6). This ensures large experimental constrains on the background uncertainties in the signal region. However, the signal over background ratio is only of the order of $30 \%$ and only 2 signal events are expected for an integrated luminosity of $\int L d t=1 \mathrm{fb}^{-1}$. An observation with a $5 \sigma$ significance would thus require an integrated luminosity of more than $80 \mathrm{fb}^{-1}$ based on pure statistical considerations. Such large integrated luminosities would require dedicated triggers for high multiplicity of low- $p_{\mathrm{T}}$ jet events during the Run-3 of the LHC, or a long data-taking period with pre-scaled jet triggers during the high luminosity phase of the LHC.

\section{Limits on instanton processes in proton-proton collisions}

\subsection{How to mimic QCD instanton signatures}

As shown in the previous sections, the most promising mass range for the observation of Instanton induced processes at the $\mathrm{LHC}$ is below $100 \mathrm{GeV}$, where the softQCD background contribution dominates. It is therefore crucial to understand if the softQCD phenomenological models have enough free- dom to mimic the QCD Instanton signatures. A first indication that this might not be easily achieved comes from the observation that the softQCD predictions from the PYTHIA, SHERPA, and the HERWIG7 generators, which implement different models, are remarkably consistent for the observables considered in this study. All of these models are however based on the same MPI description of softQCD, and with parameters tuned to describe the same, or similar data. It remains possible that with a suitable parameter choice the softQCD predictions can be made more similar to the Instanton.

As a proof-of-principle demonstration, we have tested if the softQCD Pythia predictions can be made to yield significantly more spherical events, even beyond what data indicates. Starting with the baseline MONASH tune [43] of PYTHIA8, we found that increasing the MULTIPARTONINTERACTIONS:ALPHASVALUE $=0.150$ in softQCD events does lead to more spherical events, as seen in Fig. 19. However, such a tune would also alter many other event shape distributions, such as the number of charged particles vs. $\eta$, which are not supported by data, as seen for example in Fig. 20). While the latter is based on $\sqrt{s}=7 \mathrm{TeV}$ data, the same conclusions hold for $\sqrt{s}=13 \mathrm{TeV}$. We also expect that tune of multiple parton interactions would not impact certain distributions, such as the number of tracks with displaced vertices $N_{\text {Displaced }}$, which could then be used to isolate Instanton events. As the cross section dependence on $m_{I}$ of QCD Instanton and softQCD processes is very different, it is also non-trivial to tune QCD Instanton sensitive distributions for different $m_{I}$ regions. This can be taken as a further motivation for studying Instanton production at low mass in different mass ranges, i.e. $20<m_{I}<40 \mathrm{GeV}$ and $40<m_{I}<80 \mathrm{GeV}$. We remark however that to fully rule out the potential degeneracy between more detailed studies, exploring changes to the softQCD models themselves, would be needed.

\subsection{LHC sensitivity projections}

Having defined suitable signal regions, we evaluate here the expected $95 \%$ confidence level (CL) upper limits on the instanton cross section. For each range of Instanton mass, the signal and background expectations in the respective tight signal region selection are used to perform a counting experiment using the pyhf package [56]. The systematic uncertainty on the background estimate is estimated as described in the previous sections, and ranges from about $20 \%$ at low invariant masses to about $50 \%$ at high invariant masses.

Results are shown in Fig. 21 for different assumptions on the integrated luminosity of $1 \mathrm{pb}^{-1}, 100 \mathrm{pb}^{-1}$, and $10 \mathrm{fb}^{-1}$. We can see how even with only $1 \mathrm{pb}^{-1}$, the predicted Instanton cross sections can be excluded for masses up to about $150 \mathrm{GeV}$, excluding at low masses cross sections ten times 


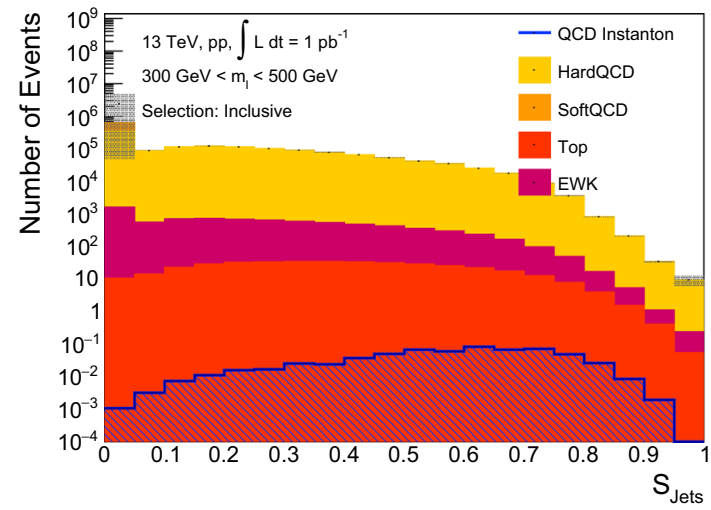

Fig. 17 Predicted distributions of the event sphericity (left) and number of reconstructed jets (right) for various processes, weighted by their predicted cross sections for an integrated luminosity of $L=\int 1 \mathrm{pb}^{-1}$. The invariant mass of all reconstructed tracks is required to be between

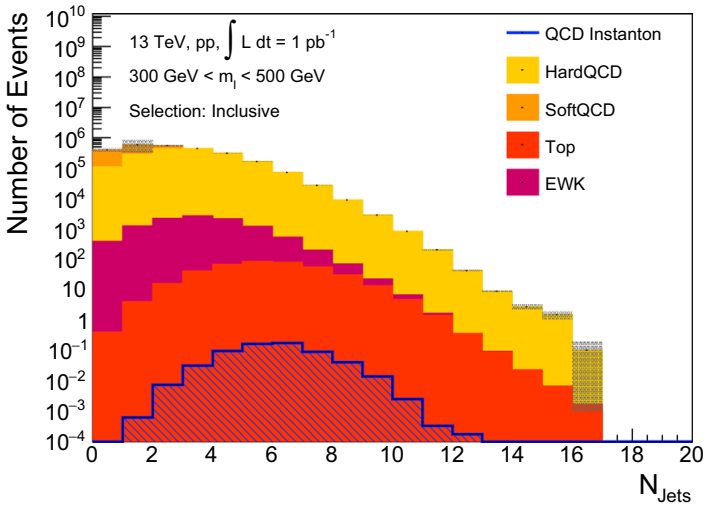

300 and $500 \mathrm{GeV}$ (high mass regime). The distributions from all processes except the Instanton process are stacked. The model uncertainties are indicated as bands

Table 6 Overview of the standard and tight signal selection as well as the definition of two control regions aiming at very low Instanton masses $\left(300 \mathrm{GeV}<m_{I}<500 \mathrm{GeV}\right)$

\begin{tabular}{|c|c|c|c|c|c|c|}
\hline & \multicolumn{3}{|c|}{$\underline{\text { Signal region }}$} & \multicolumn{3}{|c|}{ Control region } \\
\hline & Standard & Event-shape & Tight & A & $\mathrm{B}$ & $\mathrm{C}$ \\
\hline Invariant mass of rec. tracks $m_{I}$ & \multicolumn{6}{|c|}{$300 \mathrm{GeV}<m_{I}<500 \mathrm{GeV}$} \\
\hline \multicolumn{7}{|l|}{ Selection requirements } \\
\hline Number of rec. tracks, $N_{\text {Trk }}$ & - & - & - & & & \\
\hline Number of rec. tracks/inst. mass, $m_{I} / N_{\text {Trk }}$ & $<3.0$ & $<3.0$ & $<3.0$ & $>3.0$ & $<3.0$ & $<3.0$ \\
\hline Number of Jets, $N_{\text {Jets }}$ & $>7$ & $>7$ & $>7$ & $>7$ & $>7$ & $>5$ \\
\hline Broadening, $\mathcal{B}_{\text {Tracks }}$ & & $>0.3$ & $>0.3$ & $>0.3$ & $>0.3$ & $>0.3$ \\
\hline Thrust, $\mathcal{T}_{\text {Tracks }}$ & & $>0.3$ & $>0.3$ & $>0.3$ & $>0.3$ & $>0.3$ \\
\hline Number of displaced vertices, $N_{\text {Displaced }}$ & & & $>20$ & & $<15$ & $>20$ \\
\hline Identified Leptons & - & - & - & - & - & 1 \\
\hline \multicolumn{7}{|c|}{ Expected events for $\int L d t=1 \mathrm{pb}^{-1}$ in the signal region $(\mathcal{S}>0.85)$} \\
\hline$N_{\text {Signal }}$ & 0.007 & 0.004 & 0.0021 & 0.002 & 0.0003 & - \\
\hline$N_{\text {Background }}$ & 0.204 & 0.015 & 0.0074 & 33 & 0.0022 & 0.002 \\
\hline
\end{tabular}

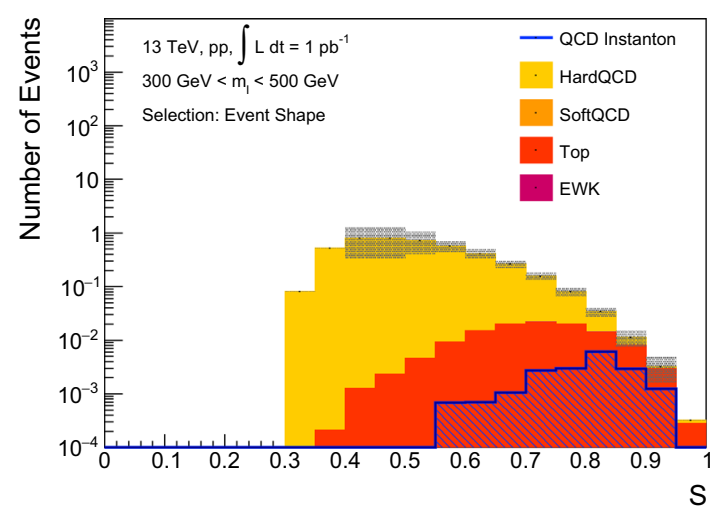

Fig. 18 Predicted distributions of the event sphericity for various processes, weighted by their predicted cross sections for an integrated luminosity of $L=\int 1 \mathrm{pb}^{-1}$ for the event-shape based selection (left) and the tight selection (right). The invariant mass of all reconstructed tracks is

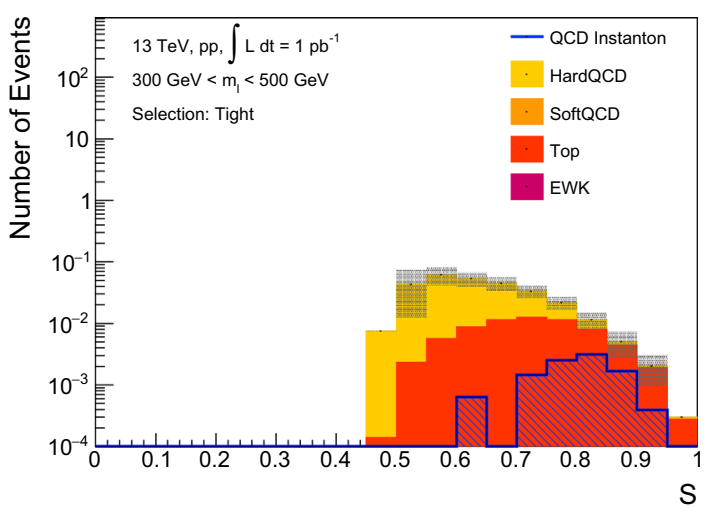

required to be between 300 and $500 \mathrm{GeV}$ (high mass regime). The distributions from all processes except the Instanton process are stacked. The model uncertainties are indicated as bands 


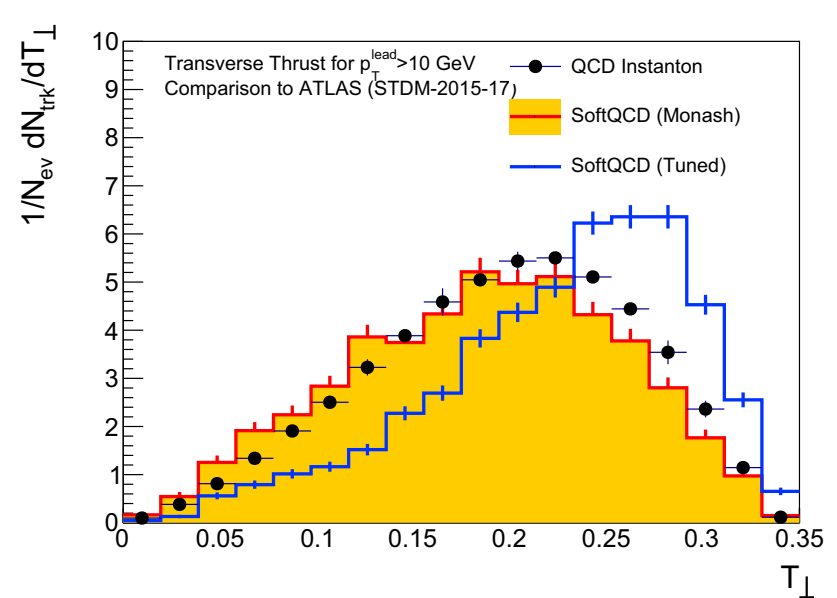

Fig. 19 Predicted distribution of the event thrust of the MONASH soft$Q C D$ tune of PYTHIA8 as well as a modified version with significantly enhanced multiple parton interaction probability (MPI:ALPHASVALUE $=0.150)$ in comparison to the measurement at $13 \mathrm{TeV}$ of the ATLAS Collaboration [54]

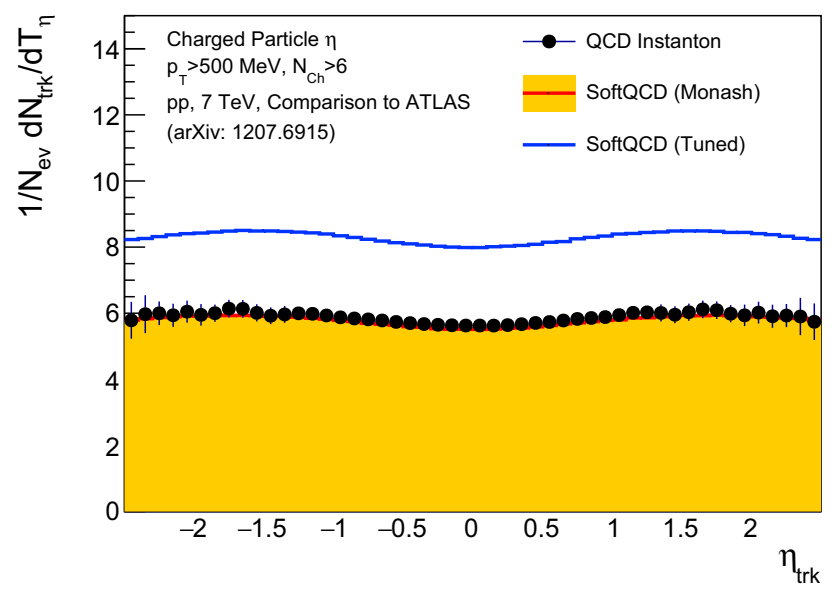

Fig. 20 Predicted distribution of the charged particle spectrum vs. $\eta$ of the MONASH softQCD tune of PYTHIA8 as well as a modified version with significantly enhanced multiple parton interaction probability (MPI:ALPHASVALUE $=0.150$ ) in comparison to the measurement at $7 \mathrm{TeV}$ of the ATLAS Collaboration [55]

smaller than those predicted by [31]. Increasing the collected luminosity to $100 \mathrm{pb}^{-1}$ would extend the limit at large invariant masses to $300 \mathrm{GeV}$, with a negligible improvement of the limit at low masses. A further integrated luminosity increase by an additional factor of 100 would push the limit at high masses beyond our last simulated mass point of $400 \mathrm{GeV}$.

\subsection{First limit on instanton processes from proton-proton collision data}

As already indicated in Fig. 8, the distribution of charged particles vs. their pseudorapidity is a sensitive observable for QCD Instanton processes in the low mass regime. This observable is routinely used in soft $Q C D$ studies and has been

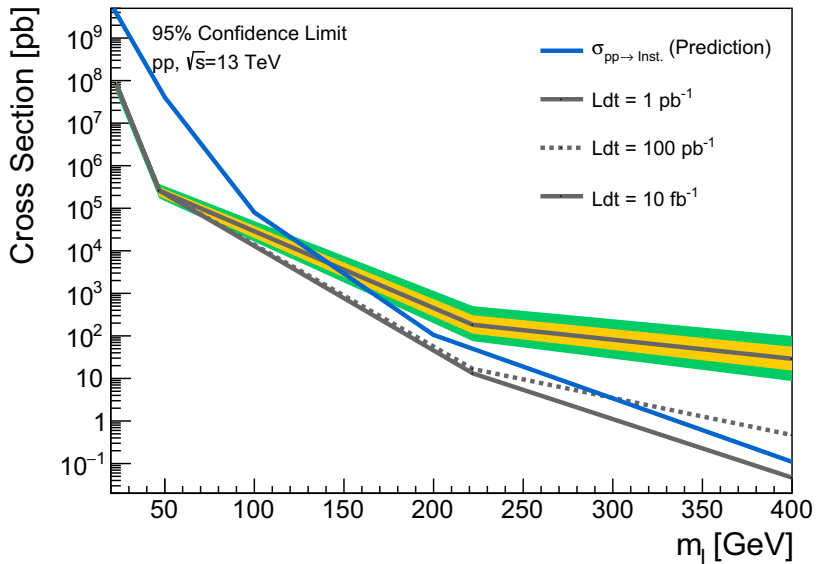

Fig. 21 Expected exclusion limits at 95\% CL on the cross section for Instanton induced processes for three different assumed integrated luminosities . Conservative systematic uncertainties on the background modelling have been made.

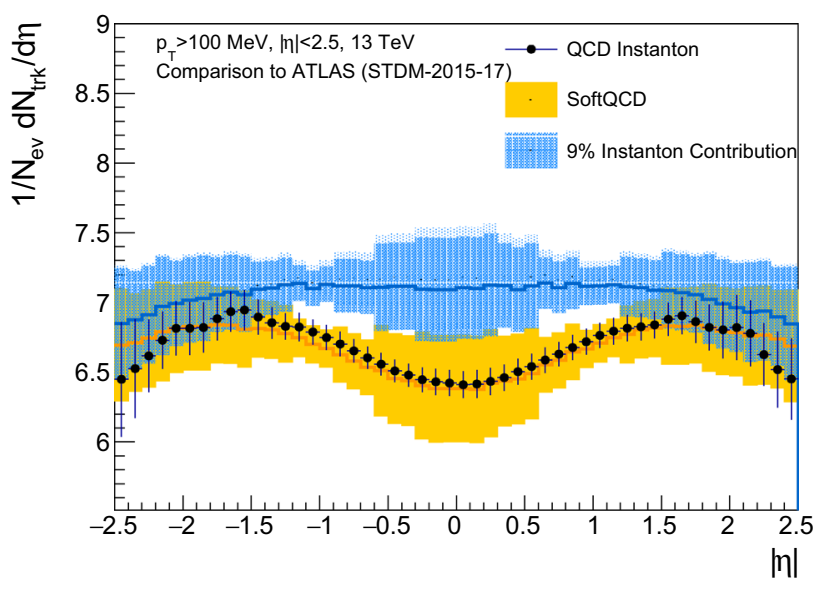

Fig. 22 Primary charged-particle multiplicities as a function of $|\eta|$ for events with at least two primary charged particles with $p_{\mathrm{T}}>100 \mathrm{MeV}$ and $|\eta|<2.5$, each with a lifetime $\tau>300 \mathrm{ps}$. The black dots represent the measurement of ATLAS [54], while the soft $Q C D$ prediction is produced with PYTHIA8. Also shown is a prediction which includes a contribution of $9 \%$ on Instanton induced processes. The model uncertainties are indicated by the bands

measured at $\sqrt{s}=13 \mathrm{TeV}$ in $p p$ collisions at the LHC $[54,57-$ 59]. We consider the ATLAS Collaboration measurement [54], which requires events with at least two primary charged particles with $p_{\mathrm{T}}>100 \mathrm{MeV}$ and $|\eta|<2.5$, each with a lifetime $\tau>300 \mathrm{ps}$, and studied to which extent these measurements can be used to constrain QCD Instanton production. The ATLAS analysis as implemented in RIVET was applied on the simulated softQCD sample based on PYTHIA8 as well as the Instanton signal sample with $s_{\min }^{\prime}>25 \mathrm{GeV}$. A modelling uncertainty on the soft $Q C D$ prediction was estimated by considering the envelope of the PYTHIA, HERWIG7 and SHERPA samples. To obtain a systematic uncertainty on the signal modelling, the differences in the $\eta_{T r k}$ distribution at 
$m_{I} \approx 500 \mathrm{GeV}$ between the SHERPA and HERWIG7 predictions have been taken as an approximation. The resulting signal and background uncertainties have been treated once fully correlated and once fully uncorrelated bin-to-bin.

The Instanton signal prediction has been added with a scaling factor $\alpha$ to the predicted soft $Q C D$ distribution, normalised accordingly and this combined prediction fitted via a $\chi^{2}$ minimization approach to the data distribution, as shown in Fig. 22. The fit yields a maximal value of $\alpha=0.09$ and $\alpha=0.03$ at $95 \% \mathrm{CL}$, assuming bin-to-bin correlated as well as bin-to-bin uncorrelated uncertainties on the predictions, respectively. The expected prediction for an Instanton contribution of $9 \%$ to the standard soft $Q C D$ processes is also shown in Fig. 22. The fiducial cross section defined by the selection $p_{\mathrm{T}}>100 \mathrm{MeV}$ and $|\eta|<2.5$, each with a lifetime $\tau>300 \mathrm{ps}$ can be estimated to be $\sigma=71 \mathrm{mb}$, when taking the integrated luminosity of $\int L d t=151 \mu \mathrm{b}^{-1}$, the number of selected events $N=9.3 \cdot 10^{6}$ and assuming a detector efficiency of $\epsilon=0.87$. Hence, an upper limit on Instanton induced processes with $s_{\min }^{\prime}>25 \mathrm{GeV}$ can be placed between 2.1 and $6.4 \mathrm{mb}$, depending on the correlation scenario assumed. In principle, further measured distributions could be used to derive more stringent limits. However, we think a dedicated analysis effort by the LHC collaborations would be the right next step to shed further light on QCD Instanton processes.

\section{Conclusion}

In this paper we presented detailed studies towards possible analysis strategies to observe Instanton induced processes in proton-proton collisions at the Large Hadron Collider. Several observables have been identified, which allow to effectively separate signal and background events. In order to study Instanton processes at higher energies, special triggers might have to be implemented for the upcoming LHC runs. However, the situation is different for low energies which have significantly larger cross sections and should be in principle already be recorded. It is concluded that the most promising phase-space region for an early observation is therefore at low energies, for Instanton masses below $100 \mathrm{GeV}$, where the cross section is very high. Since the dominant background in this energy regime is from soft$Q C D$ processes, several methods to constrain and validate softQCD models in dedicated control regions have been discussed. We find that with an integrated luminosity of just $1 \mathrm{pb}^{-1}$, the LHC can already probe this low mass Instanton regime. With $10 \mathrm{fb}^{-1}$ it would be possible to probe Instanton masses of up to $0.5 \mathrm{TeV}$. In addition, available measurements of Minimum Bias data have been used to derive a first upper limit on the cross section of Instanton processes, yielding an upper bound of $6.4 \mathrm{mb}$ for Instanton masses above $25 \mathrm{GeV}$.
The methods described in this paper will hopefully boost dedicated search efforts at the LHC over the full Instanton mass range by several experiments, leading to a robust result based on different strategies.

Acknowledgements We would like to thank Frank Krauss for all his help during the generation of Instanton signal samples with the SHERPA generator, Simon Plätzer and Andreas Papaefstathiou for providing us with a preliminary implementation of Instanton production in HERWIG7, and Daniel Milne for his help in validating our event shape calculations.

Data Availability Statement This manuscript has no associated data or the data will not be deposited. [Authors' comment: This study is of theoretical nature and has no data associated to it.]

Open Access This article is licensed under a Creative Commons Attribution 4.0 International License, which permits use, sharing, adaptation, distribution and reproduction in any medium or format, as long as you give appropriate credit to the original author(s) and the source, provide a link to the Creative Commons licence, and indicate if changes were made. The images or other third party material in this article are included in the article's Creative Commons licence, unless indicated otherwise in a credit line to the material. If material is not included in the article's Creative Commons licence and your intended use is not permitted by statutory regulation or exceeds the permitted use, you will need to obtain permission directly from the copyright holder. To view a copy of this licence, visit http://creativecomm ons.org/licenses/by/4.0/.

Funded by $\mathrm{SCOAP}^{3}$.

\section{References}

1. C.-N. Yang, R.L. Mills, Conservation of isotopic spin and isotopic gauge invariance. Phys. Rev. 96, 191-195 (1954)

2. B. Mistlberger, Higgs boson production at hadron colliders at $\mathrm{N}^{3}$ LO in QCD. JHEP 05, 028 (2018)

3. C. Duhr, F. Dulat, B. Mistlberger, Drell-Yan cross section to third order in the strong coupling constant. Phys. Rev. Lett. 125(17), 172001 (2020)

4. D. d'Enterria. Experimental QCD summary (ICHEP 2020) PoS ICHEP2020, 012 (2021). https://doi.org/10.22323/1.390.0012

5. A.A. Belavin, A.M. Polyakov, A.S. Schwartz, Y.S. Tyupkin, Pseudoparticle solutions of the Yang-Mills equations. Phys. Lett. B 59, 85-87 (1975)

6. E.V. Shuryak, The role of instantons in quantum chromodynamics. 1. Physical vacuum. Nucl. Phys. B 203, 93 (1982)

7. P.B. Arnold, L.D. McLerran, Sphalerons, small fluctuations and Baryon number violation in electroweak theory. Phys. Rev. D 36, 581 (1987)

8. H. Forkel, A primer on instantons in QCD (2000). arXiv:hep-ph/0009136

9. E. Shuryak, Lectures on nonperturbative QCD (nonperturbative topological phenomena in QCD and related theories), 12 (2018). arXiv: 1812.01509

10. V.A. Kuzmin, V.A. Rubakov, M.E. Shaposhnikov, On the anomalous electroweak Baryon number nonconservation in the early universe. Phys. Lett. B 155, 36 (1985)

11. G.R. Farrar, M.E. Shaposhnikov, Baryon asymmetry of the universe in the minimal Standard Model. Phys. Rev. Lett. 70, 2833-2836 (1993) [Erratum: Phys.Rev.Lett. 71, 210 (1993)]

12. V.A. Rubakov, M.E. Shaposhnikov, Electroweak baryon number nonconservation in the early universe and in high-energy collisions. Usp. Fiz. Nauk 166, 493-537 (1996) 
13. V.A. Rubakov, M.E. Shaposhnikov, Phys. Usp. 39, 461 (1996)

14. G. Hooft, How instantons solve the U(1) problem. Phys. Rept. 142, 357-387 (1986)

15. G. 't Hooft, Computation of the quantum effects due to a fourdimensional pseudoparticle. Phys. Rev. D 14, 3432-3450 (1976) [Erratum: Phys. Rev.D18,2199(1978)]

16. G. Hooft, Symmetry breaking through Bell-Jackiw anomalies. Phys. Rev. Lett. 37, 8-11 (1976)

17. D. Diakonov, V.Y. Petrov, A theory of light quarks in the instanton vacuum. Nucl. Phys. B 272, 457-489 (1986)

18. F.R. Klinkhamer, N.S. Manton, A saddle point solution in the Weinberg-Salam theory. Phys. Rev. D 30, 2212 (1984)

19. A. Ringwald, High-energy breakdown of perturbation theory in the electroweak instanton sector. Nucl. Phys. B 330, 1-18 (1990)

20. H. Aoyama, H. Goldberg, Anomalous Baryon number nonconservation in $\mathrm{p} p$ collisions at $40-\mathrm{TeV}$. Phys. Lett. B 188, 506-510 (1987)

21. O. Espinosa, High-energy behavior of baryon and lepton number violating scattering amplitudes and breakdown of unitarity in the standard model. Nucl. Phys. B 343, 310-340 (1990)

22. A. Ringwald, Vacuum structure and high-energy scattering. Nucl. Phys. B Proc. Suppl. 121, 145-148 (2003). https://doi.org/10.1016/ S0920-5632(03)01831-0

23. A. Ringwald, Electroweak instantons/sphalerons at VLHC? Phys. Lett. B 555, 227-237 (2003)

24. V.V. Khoze, D.L. Milne, Suppression of electroweak instanton processes in high-energy collisions. Int. J. Mod. Phys. A 36(05), 2150032 (2021). https://doi.org/10.1142/S0217751X21500329

25. S. Moch, A. Ringwald, F. Schrempp, Instantons in deep inelastic scattering: the simplest process. Nucl. Phys. B 507, 134-156 (1997)

26. A. Ringwald, F. Schrempp, Zooming in on instantons at HERA. Phys. Lett. B 503, 331-340 (2001). https://doi.org/10.1016/ S0370-2693(01)00216-7

27. A. Ringwald, F. Schrempp, QCDINS 2.0: A Monte Carlo generator for instanton induced processes in deep inelastic scattering. Comput. Phys. Commun. 132, 267-305 (2000). https://doi.org/10. 1016/S0010-4655(00)00148-X

28. C. Adloff et al., Search for QCD instanton induced processes in deep inelastic scattering at HERA. Eur. Phys. J. C 25, 495-509 (2002)

29. S. Chekanov et al., Search for QCD instanton induced events in deep inelastic ep scattering at HERA. Eur. Phys. J. C 34, 255-265 (2004)

30. V. Andreev et al., Search for QCD instanton-induced processes at HERA in the high- $Q^{2}$ domain. Eur. Phys. J. C 76(7), 381 (2016)

31. V.V. Khoze, F. Krauss, M. Schott, Large effects from small QCD instantons: making soft bombs at Hadron colliders. JHEP 04, 201 (2020)

32. V.V. Khoze, D.L. Milne, M. Spannowsky, Searching for QCD instantons at hadron colliders. Phys. Rev. D 103(1), 014017 (2021). https://doi.org/10.1103/PhysRevD.103.014017

33. A. Ringwald, F. Schrempp, Instanton induced cross-sections in deep inelastic scattering. Phys. Lett. B 438, 217-228 (1998)

34. C.W. Bernard, Gauge zero modes, instanton determinants, and QCD calculations. Phys. Rev. D 19, 3013 (1979)

35. T.R. Morris, D.A. Ross, C.T. Sachrajda, Higher order quantum corrections in the presence of an instanton background field. Nucl. Phys. B 255, 115-148 (1985)

36. A. Ringwald, F. Schrempp, Confronting instanton perturbation theory with QCD lattice results. Phys. Lett. B 459, 249-258 (1999)

37. F. Schrempp, A. Utermann, QCD instantons and high-energy diffractive scattering. Phys. Lett. B 543, 197-207 (2002)

38. A.H. Mueller, Leading power corrections to the semiclassical approximation for gauge meson collisions in the one instanton sector. Nucl. Phys. B 353, 44-58 (1991)
39. V.V. Khoze, A. Ringwald, Nonperturbative contribution to total cross-sections in nonAbelian gauge theories. Phys. Lett. B 259, 106-112 (1991)

40. R. Harnik, T. Wizansky, Signals of new physics in the underlying event. Phys. Rev. D 80, 075015 (2009)

41. J. de Favereau, C. Delaere, P. Demin, A. Giammanco, V. Lemaitre, A. Mertens, M. Selvaggi, DELPHES 3, a modular framework for fast simulation of a generic collider experiment. JHEP 02, 057 (2014)

42. T. Sjostrand, S. Mrenna, P.Z. Skands, A Brief Introduction to PYTHIA 8.1. Comput. Phys. Commun. 178, 852-867 (2008)

43. P. Skands, S. Carrazza, J. Rojo, Tuning PYTHIA 8.1: the Monash 2013 Tune. Eur. Phys. J. C 74(8), 3024 (2014)

44. R.D. Ball et al., Parton distributions with LHC data. Nucl. Phys. B 867, 244-289 (2013)

45. ATLAS Pythia 8 tunes to $7 \mathrm{TeV}$ data, Technical Report ATL-PHYSPUB-2014-021, CERN, Geneva (2014)

46. M. Bahr et al., Herwig++ physics and manual. Eur. Phys. J. C 58, 639-707 (2008)

47. E. Bothmann, et al., Event Generation with Sherpa 2.2. SciPost Phys. 7(3), 034 (2019). https://doi.org/10.21468/SciPostPhys.7.3. 034

48. E. Bothmann et al., Event generation with Sherpa 2.2. SciPost Phys. 7(3), 034 (2019)

49. R. Kleiss, W. James Stirling, S.D. Ellis, A new Monte Carlo treatment of multiparticle phase space at high-energies. Comput. Phys. Commun. 40, 359 (1986)

50. J. Bellm et al., Herwig 7.2 release note. Eur. Phys. J. C 80(5), 452 (2020)

51. M. Cacciari, G.P. Salam, G. Soyez, The anti- $k_{t}$ jet clustering algorithm. JHEP 04, 063 (2008)

52. M. Cacciari, G.P. Salam, G. Soyez, FastJet user manual. Eur. Phys. J. C 72, 1896 (2012)

53. C. Bierlich et al., Robust independent validation of experiment and theory: Rivet version 3. SciPost Phys. 8, 026 (2020)

54. M. Aaboud, et al., Charged-particle distributions at low transverse momentum in $\sqrt{s}=13 \mathrm{TeV} p p$ interactions measured with the ATLAS detector at the LHC. Eur. Phys. J. C 76(9), 502 (2016). https://doi.org/10.1140/epjc/s10052-016-4335-y

55. G. Aad, et al., Measurement of charged-particle event shape variables in $\sqrt{s}=7 \mathrm{TeV}$ proton-proton interactions with the ATLAS detector. Phys. Rev. D 88(3), 032004 (2013). https://doi.org/10. 1103/PhysRevD.88.032004

56. L. Heinrich, M. Feickert, G. Stark, K. Cranmer, pyhf: pure-Python implementation of HistFactory statistical models. J. Open Source Softw. 6(58), 2823 (2021). https://doi.org/10.21105/joss.02823

57. B. Abelev et al., Underlying event measurements in $p p$ collisions at $\sqrt{s}=0.9$ and $7 \mathrm{TeV}$ with the ALICE experiment at the LHC. JHEP 07, 116 (2012)

58. G. Aad et al., Measurement of distributions sensitive to the underlying event in inclusive Z-boson production in $p p$ collisions at $\sqrt{s}=7 \mathrm{TeV}$ with the ATLAS detector. Eur. Phys. J. C 74(12), 3195 (2014)

59. S. Chatrchyan et al., Measurement of the underlying event in the Drell-Yan process in proton-proton collisions at $\sqrt{s}=7 \mathrm{TeV}$. Eur. Phys. J. C 72, 2080 (2012) 